\title{
An adaptive optics module for deep tissue multiphoton imaging in vivo
}

\section{Na Ji ( $\nabla_{\text {jina@berkeley.edu ) }}$}

University of California, Berkeley https://orcid.org/0000-0002-5527-1663

\section{Cristina Rodriguez}

University of California, Berkeley https://orcid.org/0000-0002-5525-3174

Ichun Chen

Boston University https://orcid.org/0000-0001-6110-5285

José Rivera

University of California, Berkeley

\section{Manuel Mohr}

Stanford University https://orcid.org/0000-0002-5189-541X

\section{Yajie Liang}

University of Maryland School of Medicine

\section{Wenzhi Sun}

Chinese Institute for Brain Research

\section{Daniel Milkie}

Janelia Research Campus https://orcid.org/0000-0002-3917-6965

\section{Thomas Bifano}

Boston University

\section{Xiaoke Chen}

Stanford University

\section{Research Article}

Keywords: Compact Adaptive Optics, Fluorescence Microscopes, Tissue-induced Aberrations, Neuronal Structures, Calcium Responses

Posted Date: December 10th, 2020

DOI: https://doi.org/10.21203/rs.3.rs-115556/v1

License: (c) (i) This work is licensed under a Creative Commons Attribution 4.0 International License. Read Full License 
Version of Record: A version of this preprint was published at Nature Methods on October 4th, 2021. See the published version at https://doi.org/10.1038/s41592-021-01279-0. 


\section{An adaptive optics module for deep tissue multiphoton imaging in vivo}

Cristina Rodríguez ${ }^{1,2,11}$, Anderson Chen $2,8,11$, José A. Rivera ${ }^{1}$, Manuel A. Mohr ${ }^{3}$, Yajie Liang ${ }^{2,9}$, Wenzhi Sun 2,10 , Daniel E. Milkie $^{2}$, Thomas G. Bifano ${ }^{4,8}$, Xiaoke Chen ${ }^{3}, \mathrm{Na} \mathrm{Ji}^{1,2,5,6,7,{ }^{*}}$

Understanding complex biological systems requires visualizing structures and processes deep within living organisms. We developed a compact adaptive optics module and incorporated it into two- and three-photon fluorescence microscopes, to measure and correct tissue-induced aberrations. We resolved synaptic structures in deep cortical and subcortical areas of the mouse brain, and demonstrated high-resolution imaging of neuronal structures and somatosensory-evoked calcium responses in the mouse spinal cord at unprecedented depths in vivo.

Imaging living organisms with subcellular resolution is key for understanding biological systems. Twophoton (2P) fluorescence microscopy is an essential tool for observing cells and biological processes under physiological conditions deep inside tissue. With longer excitation wavelength experiencing reduced tissue scattering, combined with the suppression of out-of-focus excitation due to the $3^{\text {rd }}$-order nonlinear optical process, three-photon (3P) fluorescence microscopy further extends the imaging depth in opaque tissues ${ }^{1-3}$. For both $2 \mathrm{P}$ and $3 \mathrm{P}$ microscopy beyond superficial depths, however, living biological tissues aberrate the wavefront of the excitation light, leading to an enlarged excitation focus of diminished focal intensity, ultimately limiting the in vivo imaging performance to cellular resolution at depth.

In the mouse central nervous system, reliably imaging the structure and function of neurons at subcellular resolution requires that the distortion of the excitation light, accumulated as it travels through tissues, be measured and corrected by adaptive optics $(A O)$ methods ${ }^{4-7}$. How aberrations are measured is the main factor distinguishing the different implementations of $A O$ in microscopy. While direct wavefront measurement methods have high measurement speed, they require separate wavefront sensors and correctors. In addition, the introduction of exogenous near-infrared emitting dyes is required to maintain their performance in highly scattering samples such as tissues ${ }^{8,9}$. Albeit slower, indirect wavefront sensing methods are well suited for opaque media. Using two wavefront modulation devices, an indirect wavefront sensing method based on frequency multiplexed aberration measurement successfully achieved $2 \mathrm{P}$ imaging of submicrometer-sized dendritic spines in layer 5 of the mouse primary visual cortex ${ }^{10}$. However, this method was limited to a narrow band of wavelengths and slow measurement times by the liquid-crystal spatial light modulator used for aberration correction. Here, we report a compact AO module for multiphoton microscopy, composed of a novel, high-speed segmented deformable mirror (DM), two lenses, and a field stop, that overcomes the aforementioned limitations. We demonstrate high-resolution $2 \mathrm{P}$ and $3 \mathrm{P}$ fluorescence imaging in a variety of optically challenging locations in the mouse central nervous system. With $\mathrm{AO}$, we were able to resolve fine neuronal processes and synapses (e.g., dendritic spines) in deep cortical layers as well as subcortical areas of the mouse brain in vivo - features otherwise invisible without aberration correction. We further achieved highresolution in vivo imaging of neuronal structures and somatosensory-evoked calcium responses in the mouse spinal cord at depths that to our knowledge have not been reported before.

The frequency multiplexed aberration measurement ${ }^{10}$ and the subsequent correction were implemented solely by the DM (Methods). We first determined the phase gradient values to be added to each DM segment such that the beamlets reflecting off them would converge to a single point on the focal plane. To achieve this, 1Department of Physics, University of California, Berkeley, CA, USA. 2Janelia Research Campus, Howard Hughes Medical Institute, Ashburn, VA, USA. ${ }^{3}$ Department of Biology, Stanford University, Stanford, CA, USA. ${ }^{4}$ Department of Mechanical Engineering, Photonics Center, Boston University, Boston, MA, USA. ${ }^{5}$ Department of Molecular and Cell Biology, University of California, Berkeley, CA, USA. ${ }^{6}$ Helen Wills Neuroscience Institute, University of California, Berkeley, CA, USA. ${ }^{7}$ Molecular Biophysics and Integrated Bioimaging Division, Lawrence Berkeley National Laboratory, Berkeley, CA, USA. ${ }^{8}$ Present address: Bio Optical \& Acoustic Spectroscopy Lab, Neurophotonics Center, Boston University, Boston, MA, USA. ${ }^{9}$ Present address: Department of Diagnostic Radiology and Nuclear Medicine, University of Maryland School of Medicine, Baltimore, MD, USA. ${ }^{10}$ Present address: School of Basic Medical Sciences, Capital Medical University, Beijing, China; and Chinese Institute for Brain Research, Beijing, China. ${ }^{11}$ These authors contributed equally to this work: Cristina Rodríguez, Anderson Chen. "Corresponding author: jina@berkeley.edu 
we formed a stationary reference focus using half of the beamlets and raster-scanned the other half of the beamlets around this focus by tip-tilting the corresponding DM segments. At each set of phase gradients (i.e., produced by tipping and tilting the mirror segments leading to beamlet displacements on the focal plane), we recorded the fluorescence signal variation while modulating the phase or intensity of the scanned beamlets at distinct $\sim \mathrm{kHz}$ frequencies using the DM segments. The interference strength of each beamlet with the reference focus was obtained by Fourier transforming the signal trace and recording the Fourier magnitude at its modulation frequency. The phase gradients giving rise to maximal interference strength for these beamlets were then calculated. Swapping the scanned and the stationary DM segments and repeating the phase gradient measurement, we obtained the wavefront gradients required to converge all beamlets to the same focus. We then measured the phase of each beamlet directly following a similar modulation-based procedure ${ }^{11}$, again taking advantage of the high-speed DM segments. For larger aberrations, the whole procedure may be repeated for several iterations to achieve optimal aberration correction. The final corrective wavefront was then applied to the DM for aberration correction. After reflecting off the DM and traveling through the sample, all beamlets converged around a common spot with the same phase so that they constructively interfered to form a diffraction-limited focus. In contrast to previous work ${ }^{10}$, where a slow DM or digital micromirror device was used for frequency multiplexed modulation, and a spatial light modulator for aberration measurement and correction, using a single high-speed DM both simplifies our module and enables faster $(\sim 4 \times)$ measurement time, high power throughput, along with polarization- and wavelength-independent operation, which allowed us to use the same module for $2 \mathrm{P}$ and $3 \mathrm{P}$ fluorescence microscopy.

We incorporated our $\mathrm{AO}$ module into a homebuilt 2P fluorescence microscope by placing it between the excitation laser and the microscope (Supplementary Fig. 1a). We first validated its performance in correcting artificial aberrations using the signal from fluorescent features of different sizes. For one representative artificial aberration (Supplementary Fig. 2), both intensity and phase modulation successfully enabled corrections that substantially increased signal and improved resolution of fluorescent bead images, with higher signal recovery for smaller beads $(23.7 \times$ for $0.5-\mu \mathrm{m}$-diameter bead versus $3.7 \times$ for $10-\mu \mathrm{m}$-diameter bead). We next applied the AO module to high-resolution in vivo imaging. In one example, we imaged GFP-labeled myotomes in the midtrunk of a young zebrafish larva, using a $920 \mathrm{~nm}$ laser excitation wavelength (Fig. 1a-C). At an imaging depth of $110 \mu \mathrm{m}$ below the surface, the aberration introduced by this sample, mostly astigmatism resulting from the larva's highly curved cylindrical-like surface, led to images of low resolution and contrast (Fig. 1a). Aberration correction resulted in a $\sim 2$-fold increase of $2 \mathrm{P}$ fluorescence signal and most notably, a substantial $(\sim 3.6 \times)$ improvement in image contrast.
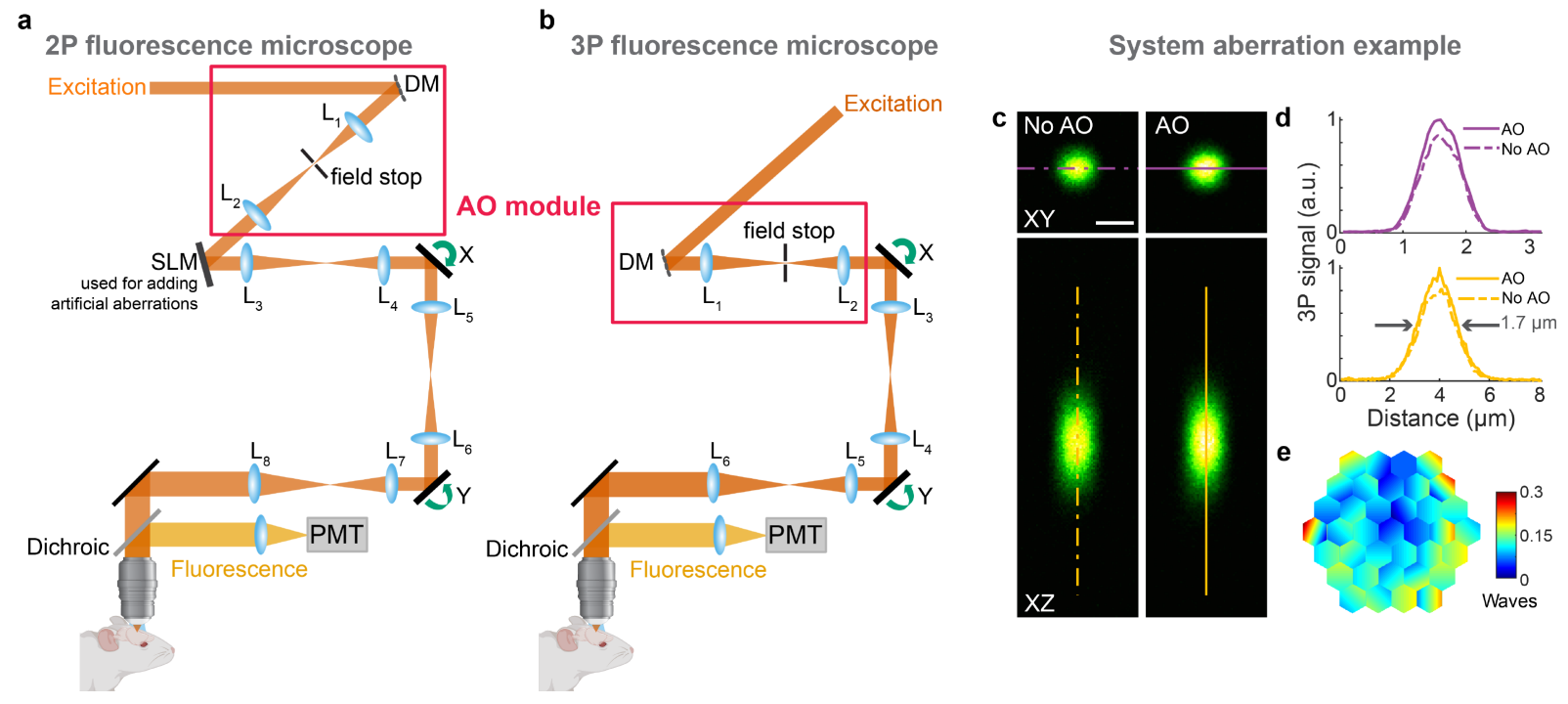

Supplementary Figure 1 | Schematics of AO 2P and 3P fluorescence microscopes, and example system correction. a, b, Components of $\mathrm{AO} 2 \mathrm{P}$ and $3 \mathrm{P}$ fluorescence microscopes, respectively. DM, deformable mirror; SLM, spatial light modulator (used to introduce artificial aberration); L, lenses; $X$ and $Y$, galvanometers; PMT, photomultiplier tube. c, Lateral and axial 3P images of a 1- $\mu$ m- 

along the purple and yellow lines in c. e, Corrective wavefront applied to the DM. Scale bar, $1 \mu \mathrm{m}$. Microscope objective: NA $1.0525 \times$.

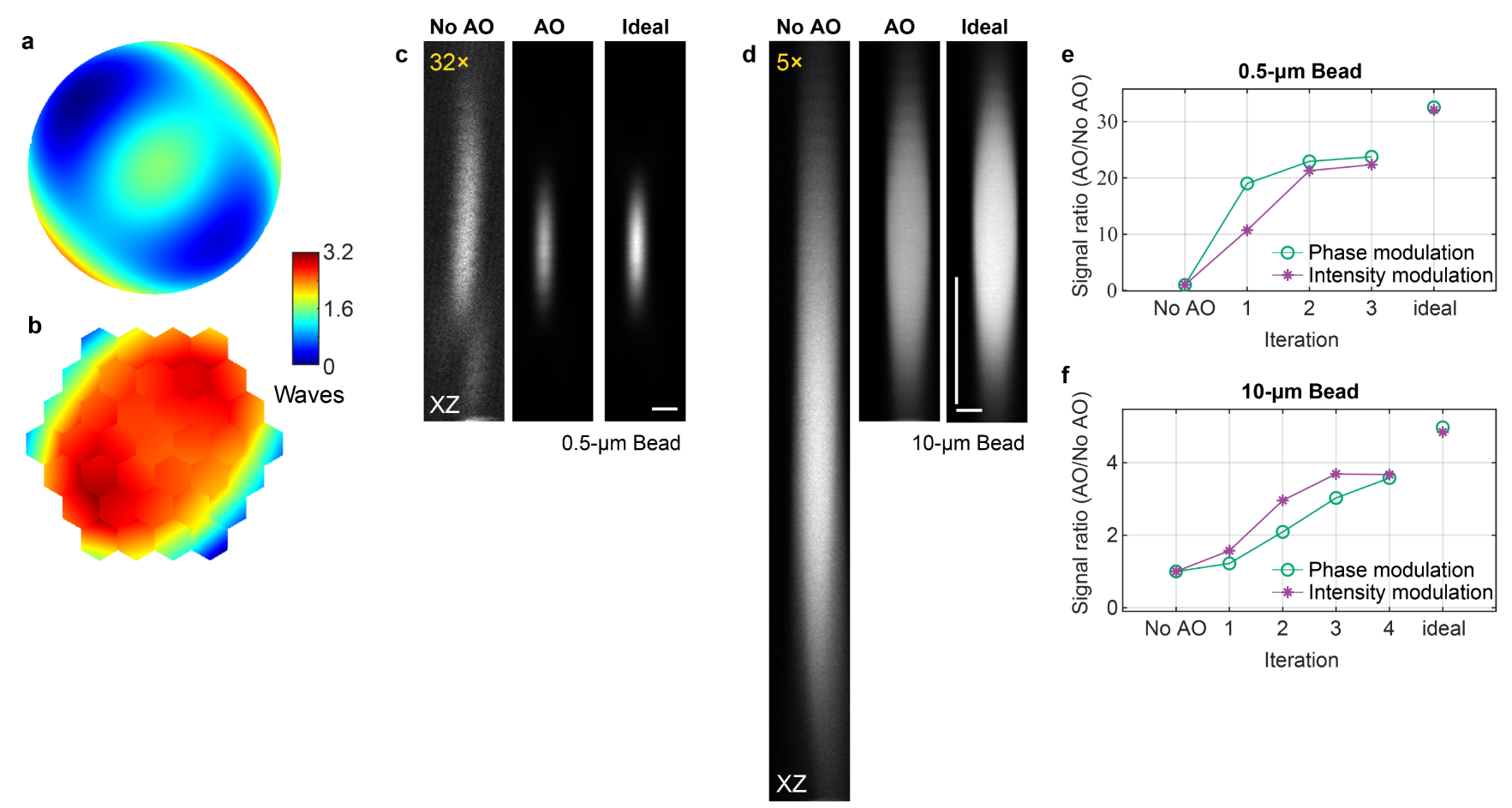

Supplementary Figure 2 | Correcting artificial aberrations for $2 \mathrm{P}$ microscopy using phase versus intensity modulation and signal from fluorescent features of different sizes. a,b. Artificial aberration introduced with the SLM and corrective wavefront on the $\mathrm{DM}$, respectively. c,d, Axial images of $0.5-\mu \mathrm{m}$ - and $10-\mu \mathrm{m}$-diameter fluorescent beads, respectively, without $\mathrm{AO}$, with $\mathrm{AO}$ (phase modulation), and under ideal aberration-free conditions (without artificial aberrations applied to the SLM). Digital gains were applied to No AO images to increase visibility. Post-objective power: 6 and $2.3 \mathrm{~mW}$, respectively. e,f, 2P signal versus iteration number for $0.5-$

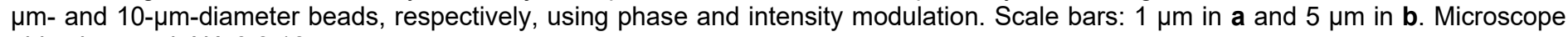
objective used: NA $0.816 \times$.

Substantial improvements in image quality were also achieved for in vivo $2 \mathrm{P}$ imaging of the strongly scattering mouse brain through a cranial window. At imaging depths of 370 and $500 \mu \mathrm{m}$ below dura, aberration correction improved the image signal and contrast of YFP-labeled dendrites (Fig. 1d-i), enabling the identification of fine features such as dendritic spines and axonal boutons - which in some cases can only be resolved in the aberration-corrected image (Fig. 1g,h). Here, and in the examples that follow, the non-corrected ("No AO") images were taken after correcting aberrations intrinsic to the microscope system (Supplementary Fig. 1c-e) and adjusting the objective correction collar to remove the spherical aberration added by the coverglass used in sample preparation (Methods). These "No AO" images, therefore, represent the best performance that conventional optics and best practice can achieve. The improvements in signal, resolution, and contrast therefore only arose from $\mathrm{AO}$ correction of tissue-induced aberrations. 


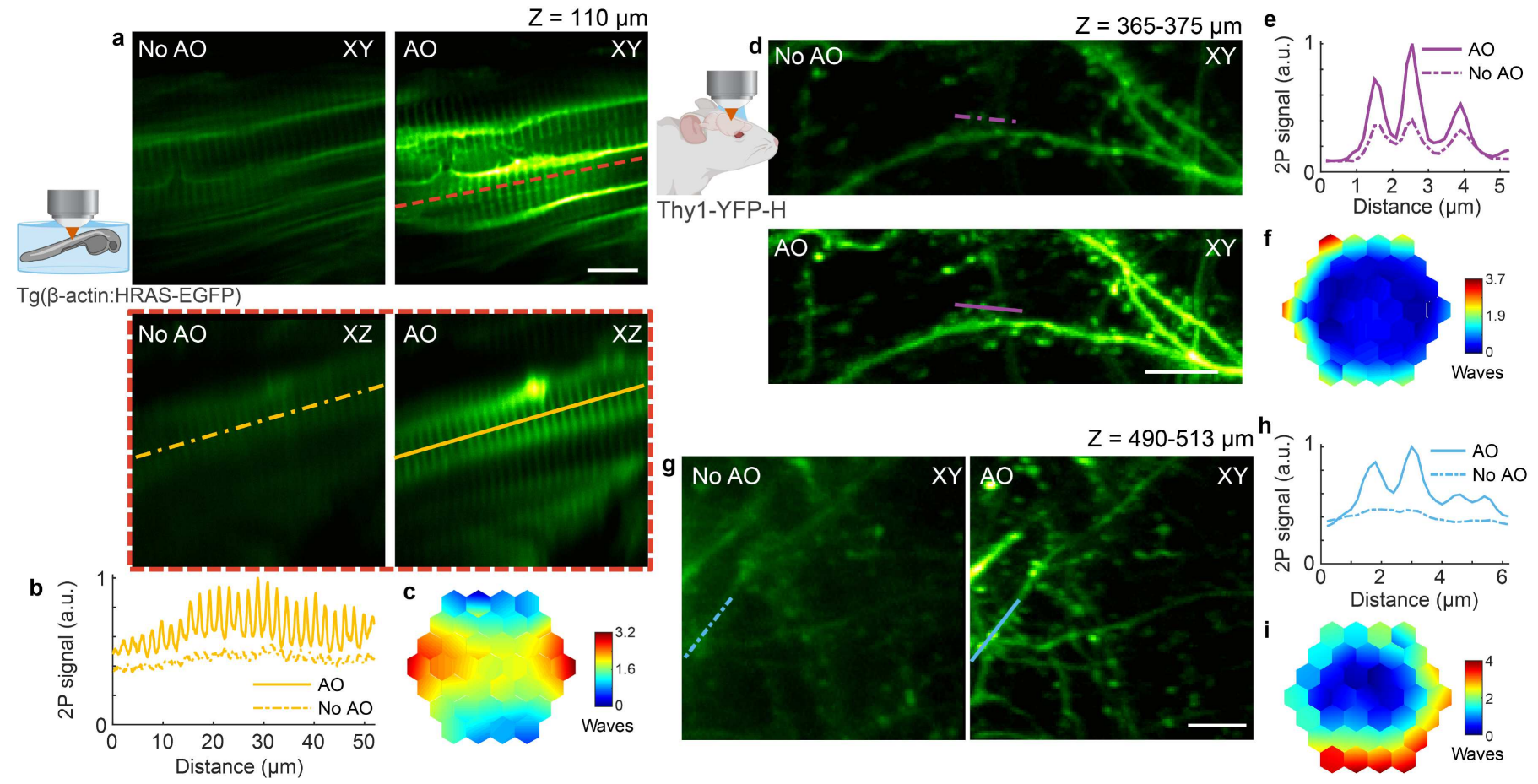

Fig. 1 | AO improves in vivo 2P imaging of myotomes in zebrafish larva and neuronal structures in the mouse brain. a, Lateral and axial (along red dashed line) images of myotomes in the mid-trunk of a 4-day-old zebrafish larva, at an imaging depth of $110 \mu \mathrm{m}$ from the surface, without and with AO (phase modulation). Post-objective power: $13 \mathrm{~mW}$. b. Signal profiles in the axial plane along the yellow lines in a. c, Corrective wavefront in a. $\mathbf{d}, \mathbf{g}$, Maximum intensity projections of dendrites in the Thy1-YFP-H mouse cerebral cortex at 365-375 $\mu \mathrm{m}$ and 490-513 $\mu \mathrm{m}$ below dura, respectively, under $920 \mathrm{~nm}$ excitation, without and with AO (phase modulation). Postobjective power: 31 and $128 \mathrm{~mW}$, respectively. e,h, Signal profiles along the purple and blue lines in $\mathbf{d}$ and $\mathbf{g}$, respectively. f,i, Corrective wavefronts in $\mathbf{d}$ and $\mathbf{g}$, respectively. Scale bars, $10 \mu \mathrm{m}$ in $\mathbf{a}$ and $5 \mu \mathrm{m}$ in $\mathbf{d}$ and $\mathbf{g}$. Microscope objective: NA $0.816 \times$ for a and NA $1.0525 \times$ for $\mathbf{d}$ and $\mathbf{g}$.

To push the imaging depth even further, we incorporated our AO module into a homebuilt 3P microscope (Supplementary Fig. 1b). After validating its ability to recover nearly-diffraction limited imaging performance under large wavefront aberrations (e.g., beads in a capillary tube, 270x signal improvement, Supplementary Fig. 3), we imaged neuronal structures throughout the mouse cerebral cortex in vivo. As an example, at 1300 $\mathrm{nm}$ excitation, a single correction pattern applied to the DM drastically improved the image quality of YFPlabeled neurons (Fig. 2a-d, Supplementary Fig. 4, and Supplementary Videos 1 and 2), located $760 \mu \mathrm{m}$ below dura. Here, AO correction led to 3P signal increases ranging from 7-fold on the cell body to 19-fold on dendrites (Fig. 2c). Moreover, previously invisible synaptic structures (e.g., dendritic spines and spine necks) became easily detectable only after aberration correction (Fig. 2b). It should be noted that, in all mouse brain examples shown here, only 2-3 rounds of correction were used to obtain the final corrective wavefront. Additional rounds did not result in substantial improvement of the fluorescence signal (Supplementary Fig. 5). The data shown is representative of several imaging sessions performed at similar depths (600-870 $\mu \mathrm{m}$ below dura), in different regions of the same animal, as well as in several animals ( $N=7)$, and under different labelling conditions (Supplementary Figs. 6-8 and Supplementary Videos 3 and 4). Aberration correction consistently improved the image signal and resolution, allowing us to resolve synaptic structures down to $870 \mu \mathrm{m}$ below dura (Supplementary Fig. 8), which were otherwise not identifiable without correction. 
Iteration

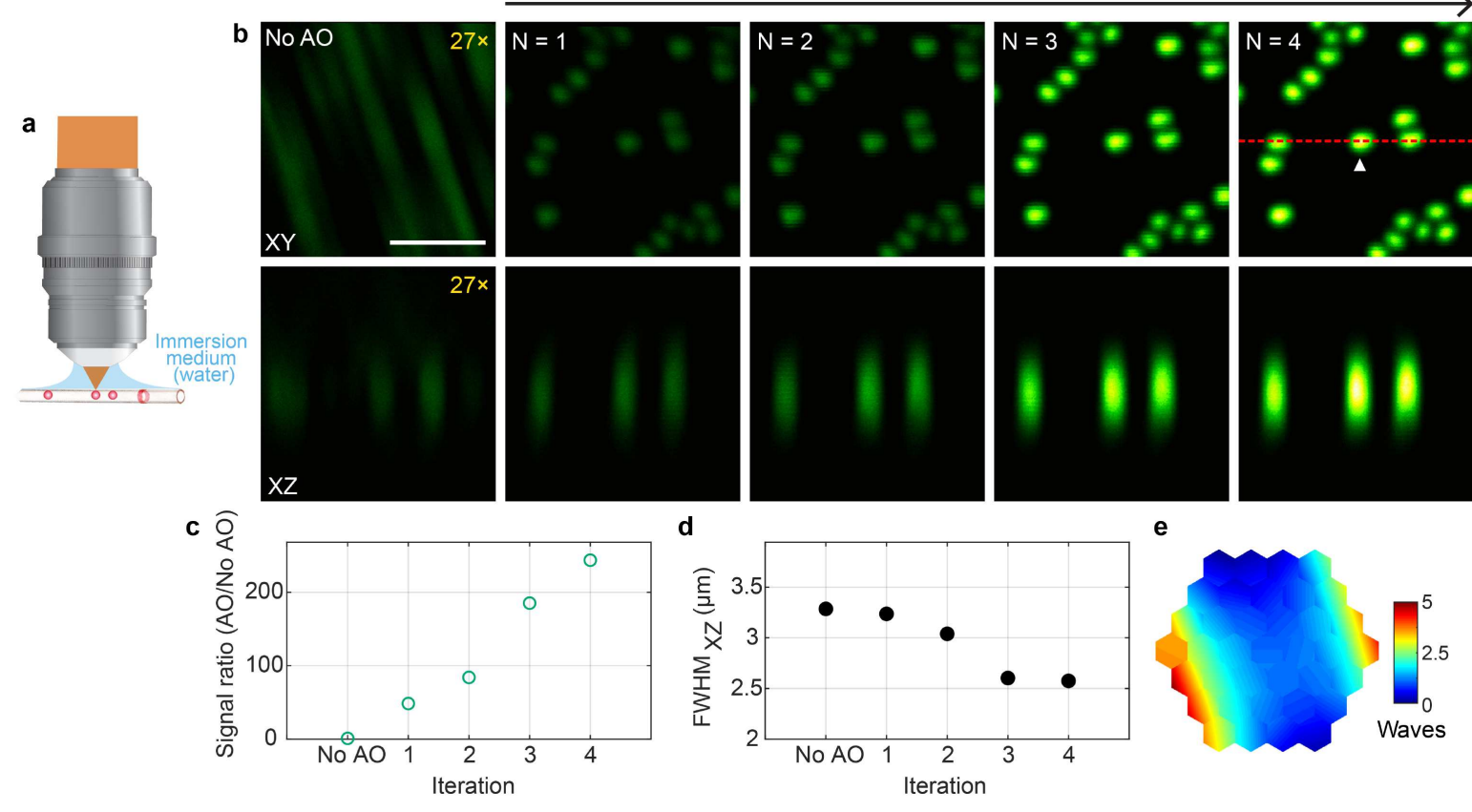

Supplementary Figure 3 | AO improves 3P imaging of beads in a capillary tube. a, Schematics of sample geometry of 1- $\mu$ mdiameter fluorescent beads in an air-filled capillary tube. b, Lateral and axial (along red dotted line) images of beads without and with AO (phase modulation). Post-objective power: $0.13 \mathrm{~mW}$. Digital gains were applied to No AO images to increase visibility. c,d, $3 \mathrm{P}$ signal improvement (AO/No AO) and axial full width at half maximum (FWHM) of a representative bead (white arrowhead in b) as a function of the iteration \#, respectively. e, Corrective wavefront. Scale bar, $5 \mu \mathrm{m}$. Microscope objective: NA $1.0525 \times$.

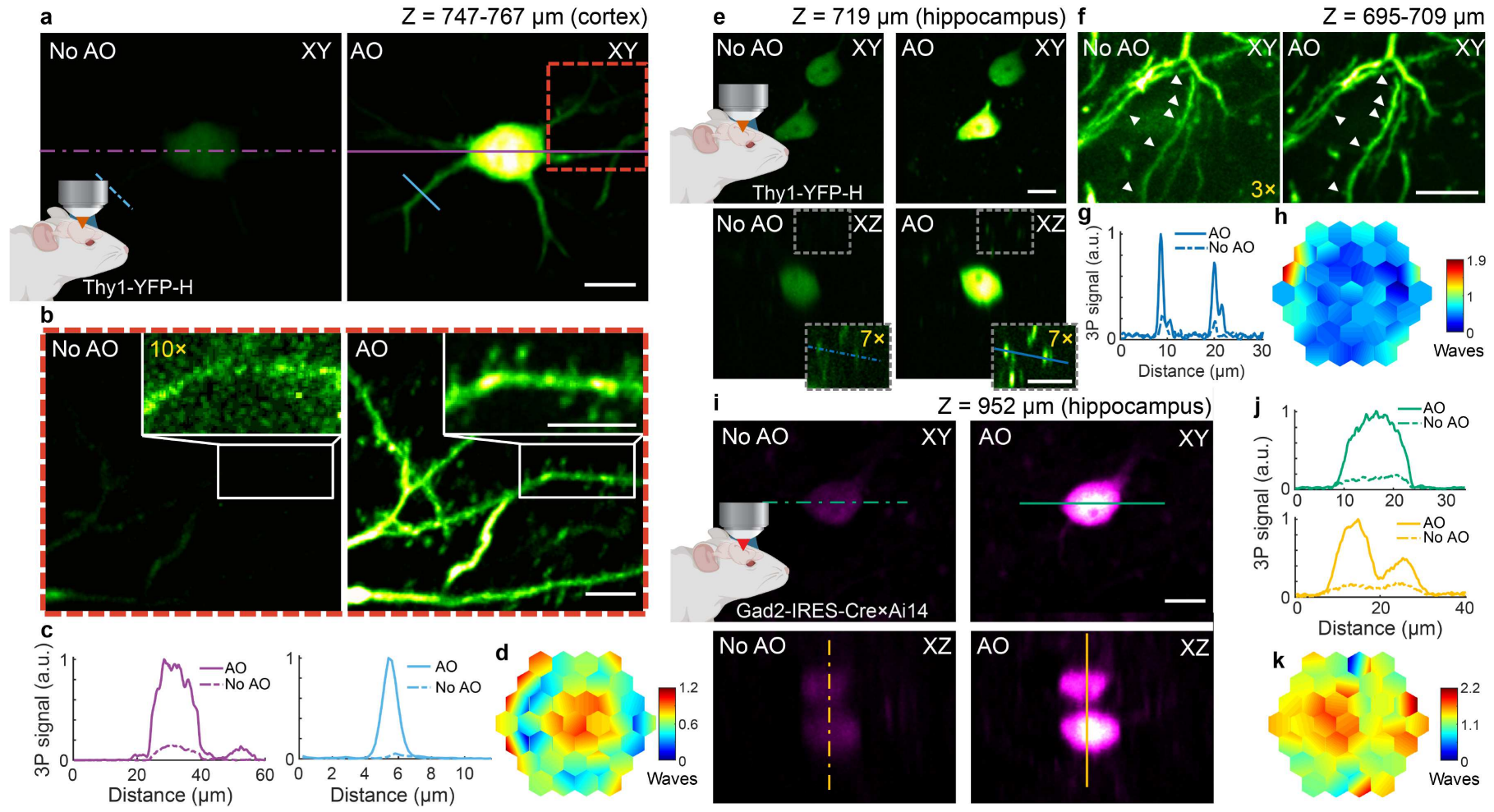

Fig. 2 | AO enables in vivo 3P imaging of cortical and hippocampal neuronal structures in the mouse brain, with subcellular resolution. a, Maximum intensity projection (MIP) of a neuron in the mouse cortex (Thy1-YFP-H), at 747-767 $\mu \mathrm{m}$ below dura, under $1300 \mathrm{~nm}$ excitation, without and with AO (phase modulation). Post-objective power: $17 \mathrm{~mW}$. b, Zoomed-in views of the red square in a, at 751-767 $\mu \mathrm{m}$ below dura, without and with AO. Insets, zoomed-in views of the dendrite in white rectangles in $\mathbf{b}$. 10× digital gain was applied to No AO inset to increase visibility. Post-objective power: $20 \mathrm{~mW}$. c. Signal profiles along the purple and blue lines in a. d, Corrective wavefront in $\mathbf{a}$ and $\mathbf{b}$. e, Lateral and axial images of neurons in the mouse hippocampus (Thy1-YFP-H), $719 \mu \mathrm{m}$ below dura, under $1300 \mathrm{~nm}$ excitation, without and with AO (phase modulation). Post-objective power: $16 \mathrm{~mW}$. Insets, zoomed-in views of the gray square in e. $7 \times$ digital gain was applied to increase visibility. f, MIP of neuronal processes above the cell body in e, at $695-709 \mu \mathrm{m}$ below dura, without and with AO. White arrows: dendritic spines. Post-objective power: $26 \mathrm{~mW}$. $3 \times$ digital gain was applied to image without AO to improve visibility. $\mathbf{g}$, Signal profiles along the blue lines in $\mathbf{e} . \mathbf{h}$, Corrective wavefront in $\mathbf{e}$ and $\mathbf{f}$. $\mathbf{i}$, Lateral and axial images 
of neurons in the mouse hippocampus (Gad2-IRES-Cre $\times$ Ai14 (Rosa26-CAG-LSL-tdTomato)), $952 \mu \mathrm{m}$ below dura, under $1700 \mathrm{~nm}$ excitation, without and with $\mathrm{AO}$ (phase modulation). Post-objective power: $30 \mathrm{~mW}$. j, Signal profiles along the green and yellow lines in i. k, Corrective wavefront in i. Scale bars, $10 \mu \mathrm{m}$. Microscope objective: NA 1.05 25x.
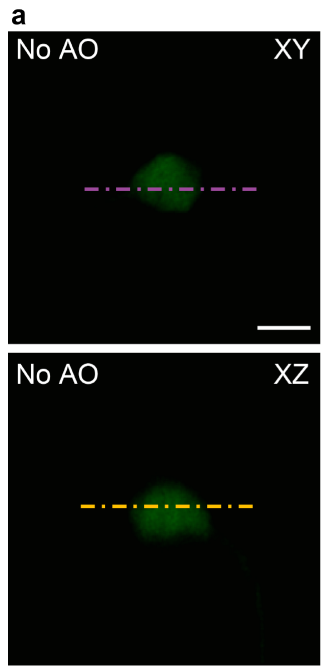

$Z=757 \mu \mathrm{m}$
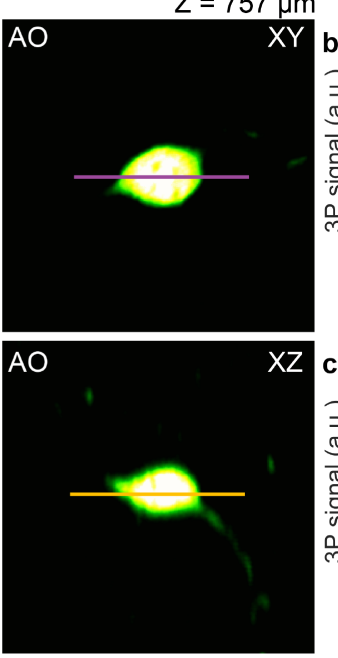

d
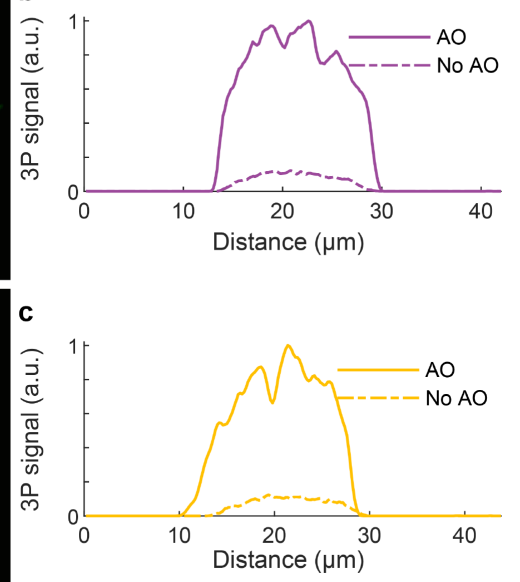

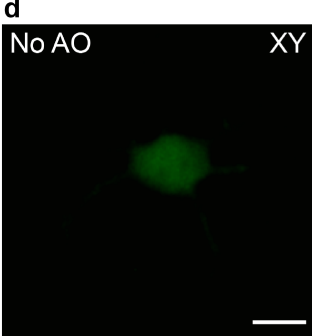

e

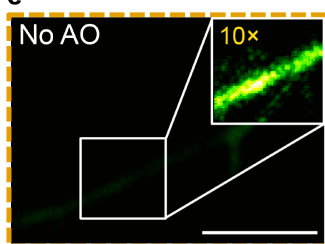

$Z=747-767 \mu \mathrm{m}$

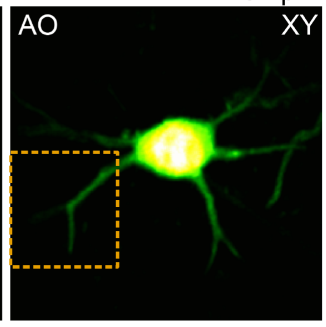

$Z=747-757 \mu m$

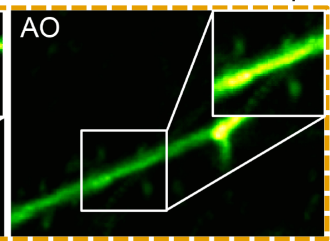

Supplementary Figure 4 | AO improves in vivo 3P imaging of cortical neurons in the mouse brain (same cell body as in Fig. 2a). a, Lateral and axial images of a neuronal cell body (Thy1-YFP-H), at $757 \mu \mathrm{m}$ below dura, under $1300 \mathrm{~nm}$ excitation, without and with AO. Post-objective power: $17 \mathrm{~mW}$. b,c, Signal profiles along the purple and yellow lines in a. d, Maximum intensity projection (MIP) of same neuron as in a, 747-767 $\mu \mathrm{m}$ below dura, under $1300 \mathrm{~nm}$ excitation, without and with AO. Post-objective power: $17 \mathrm{~mW}$. e, MIP of the yellow square in d, at 747-757 $\mu \mathrm{m}$ below dura, without and with AO. Insets, zoomed-in views of dendrite in white box. 10x digital gain was applied to the inset without AO to improve visibility. Post-objective power: $20 \mathrm{~mW}$. Scale bar, $10 \mu \mathrm{m}$. Microscope objective: NA $1.0525 \times$.

Phase modulation
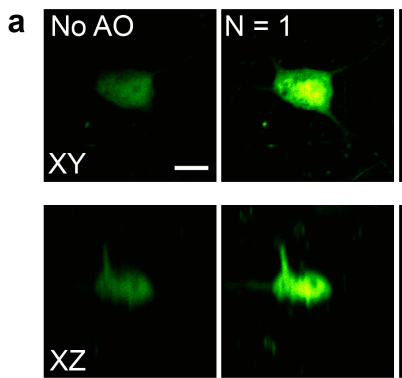

Intensity modulation
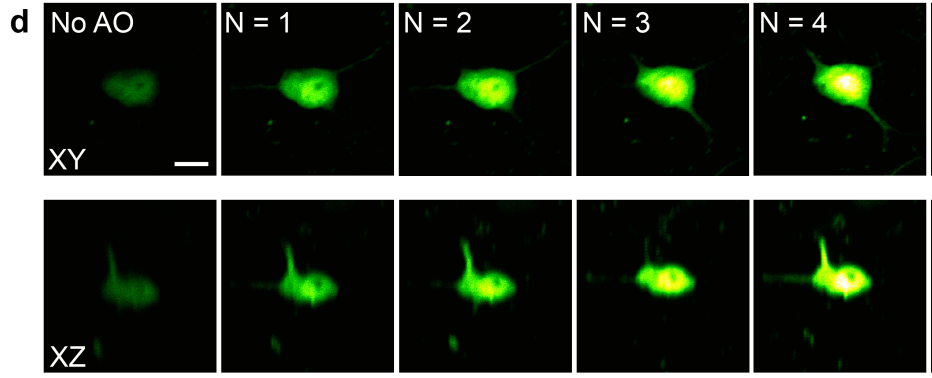

Iteration

$$
Z=623 \mu \mathrm{m}
$$
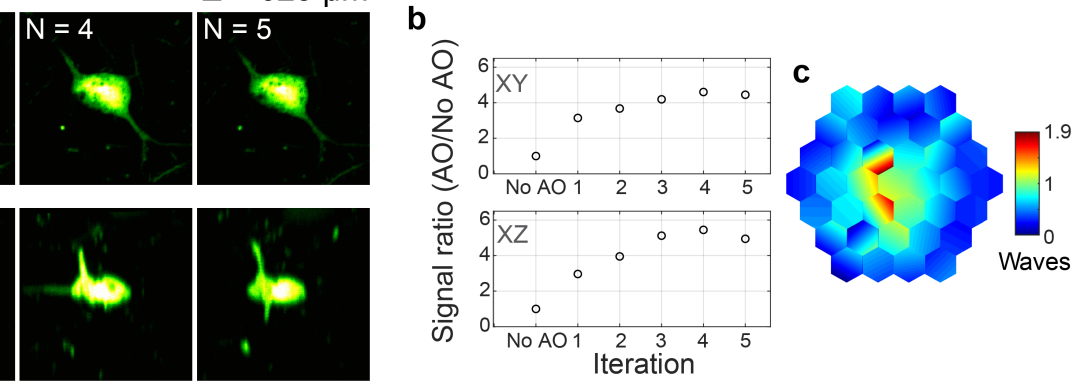

Iteration

\section{e}
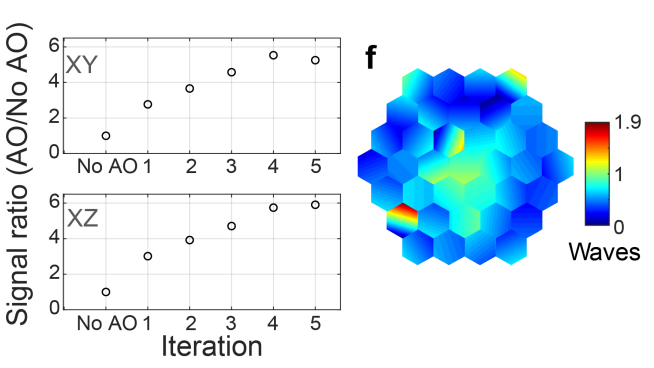

Supplementary Figure 5 | Effect of iterations on 3P fluorescence signal improvement for phase and intensity modulationbased aberration correction in the mouse brain in vivo. a,d, Lateral and axial images of a neuron in the mouse cortex (Thy1-YFP$\mathrm{H}), 623 \mu \mathrm{m}$ below dura, under $1300 \mathrm{~nm}$ excitation, without $\mathrm{AO}$ correction and after running aberration measurement a total of $\mathrm{N}=1-5$ iterations, using phase and intensity modulation, respectively. Post-objective power: 20.8 and $23.6 \mathrm{~mW}$, in a and d respectively. b, e, $3 \mathrm{P}$ signal improvement ( $\mathrm{AO} / \mathrm{No} \mathrm{AO})$ with iterations, for phase and intensity modulation, respectively. The plotted signal is the average pixel intensity within a $16 \times 16$-pixel area around the image maximum. c,f, Corrective wavefronts measured with phase and amplitude modulation, respectively. Scale bars, $10 \mu \mathrm{m}$. Microscope objective: NA $1.0525 \times$. 

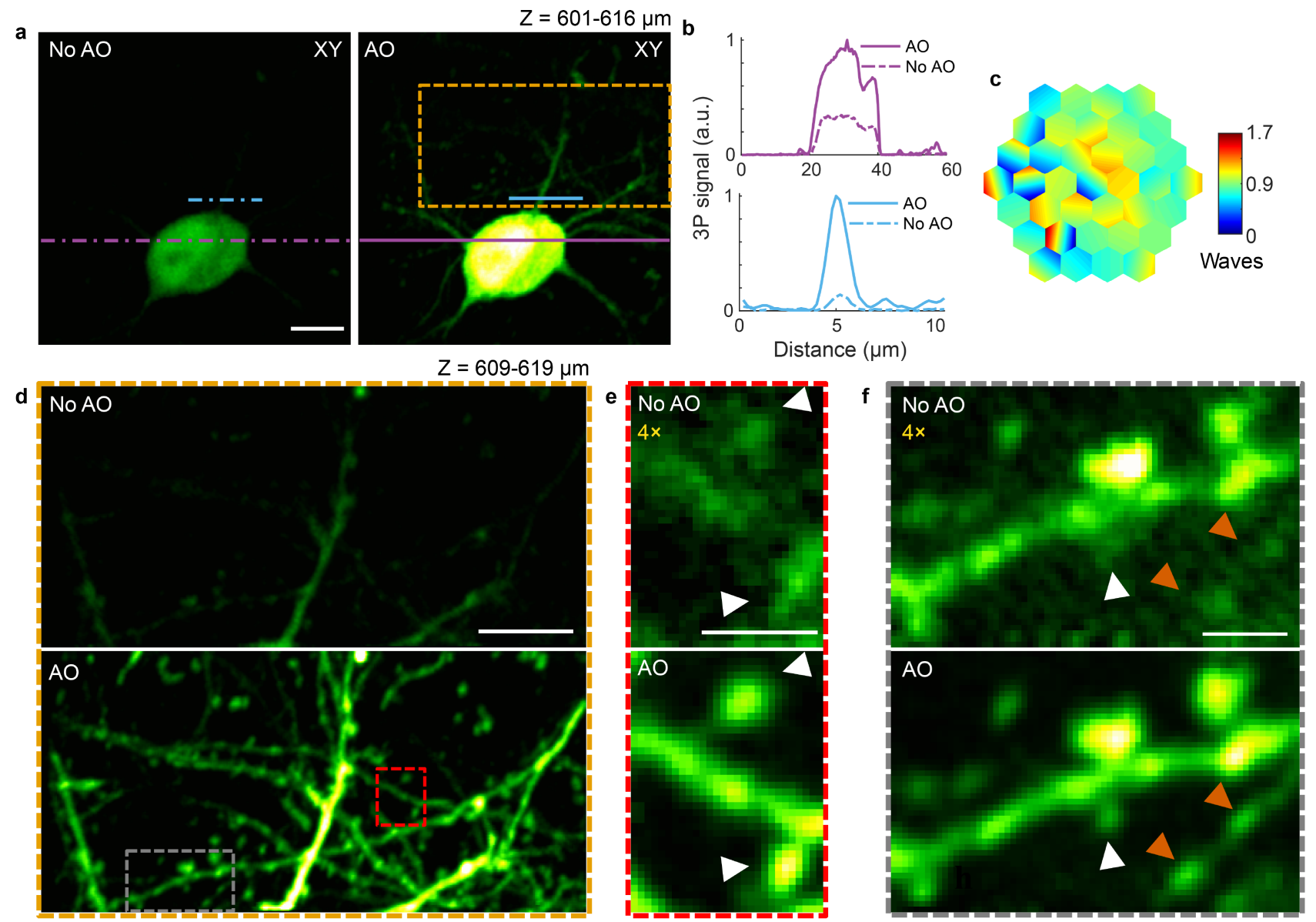

Supplementary Figure 6 | AO enables in vivo 3P imaging of dendritic spines and axonal boutons in deep layers of the mouse cortex. a, Maximum intensity projection (MIP) of a neuron in the mouse cortex (Thy1-YFP-H), at $601-616 \mu \mathrm{m}$ below dura, under 1300 $\mathrm{nm}$ excitation, without and with AO. Post-objective power: $17 \mathrm{~mW}$. b. Signal profiles along the purple and blue lines in a. c, Corrective wavefront in a. d, MIP of the orange box in a, at 609-619 $\mu \mathrm{m}$ below dura, without and with AO. Post-objective power: $25.6 \mathrm{~mW}$. e,f, Zoomed-in views of the red and gray boxes in $\mathbf{d}$, respectively. $4 \times$ digital gain was applied to images without AO to improve visibility. White arrowheads: dendritic spines; orange arrowheads: axonal boutons. Scale bars: $10 \mu \mathrm{m}$ in a and d; $2 \mu \mathrm{m}$ in e and f. Microscope objective: NA $1.0525 \times$.
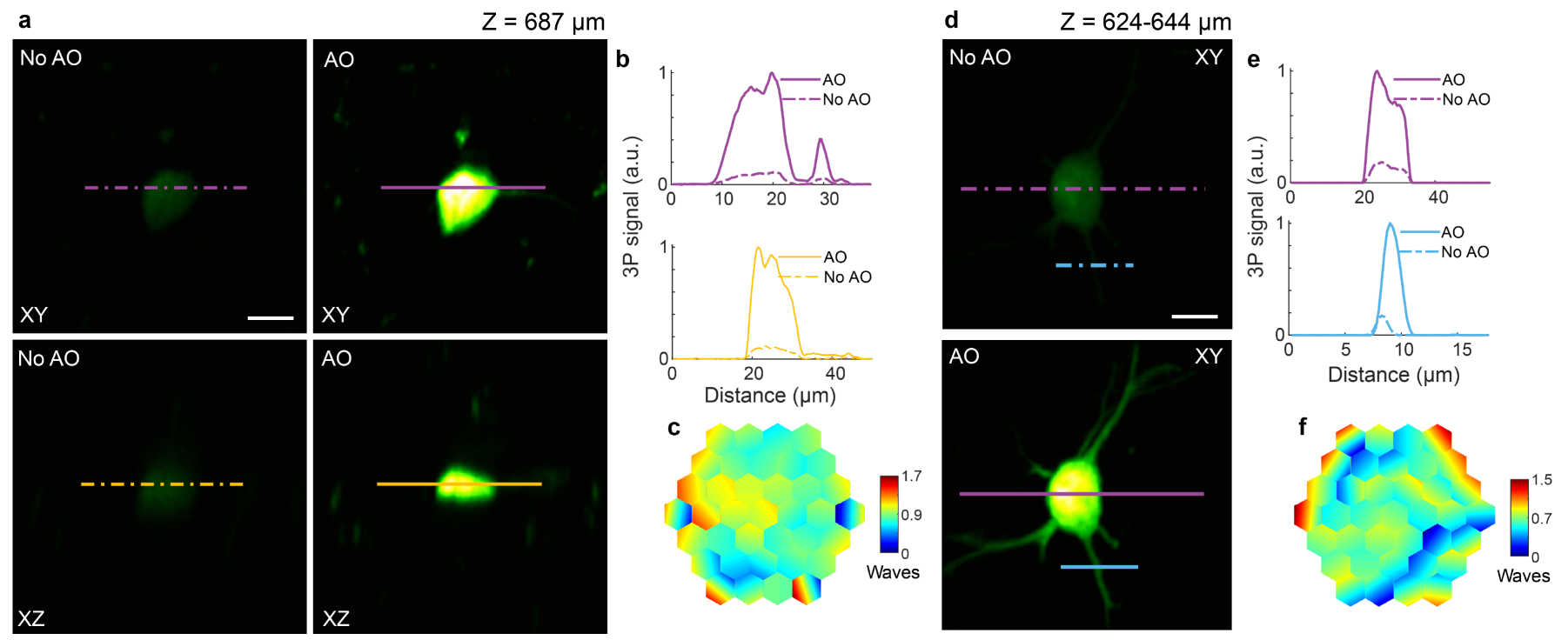

Supplementary Figure 7 | AO improves in vivo 3P imaging of cortical neuronal structures in the brain of a Thy1-GFP-M mouse. a, Lateral and axial images of a neuron in the mouse cortex (Thy1-GFP-M), at $687 \mu \mathrm{m}$ below dura, under $1300 \mathrm{~nm}$ excitation, taken without and with AO. Post-objective power: $35 \mathrm{~mW}$. b. Signal profiles along the purple and yellow lines in a. c, Corrective wavefront in a. d, Maximum intensity projection of a neuron in the mouse cortex (Thy1-GFP line M, different animal than in a), at $624-644 \mu \mathrm{m}$ below dura, under $1300 \mathrm{~nm}$ excitation, without and with AO. Post-objective power: $13 \mathrm{~mW}$. e, Signal profiles along the purple and blue lines in d. f, Corrective wavefront in d. Scale bars, $10 \mu \mathrm{m}$. Microscope objective: NA 1.05 25×. 

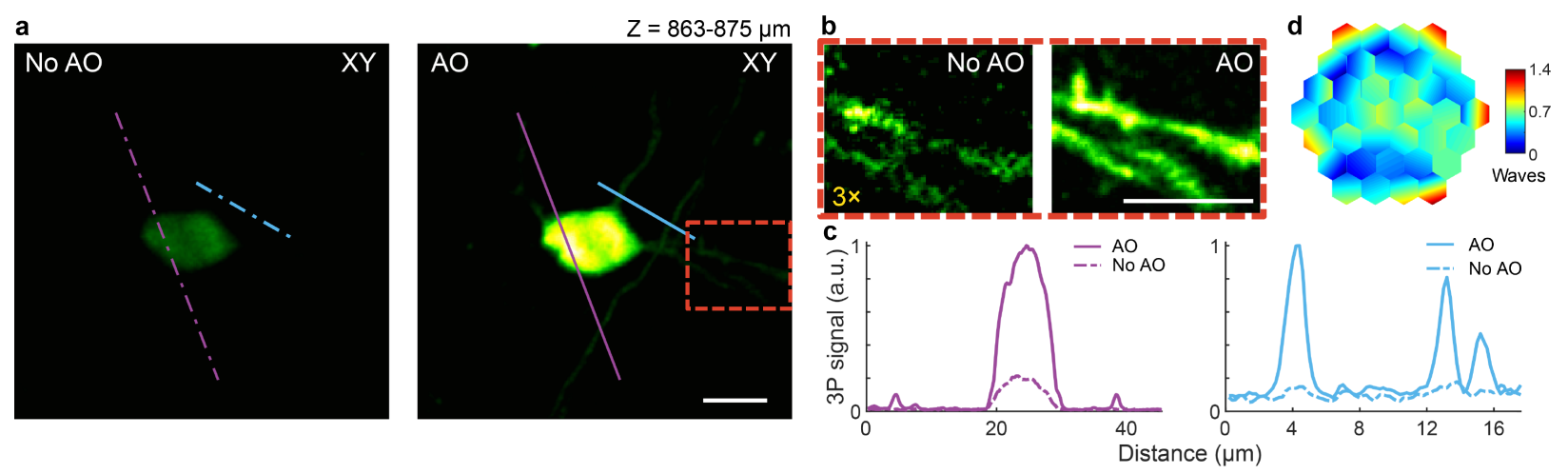

Supplementary Figure 8 | AO enables in vivo 3P imaging of synapses in the mouse cortex, $869 \mu \mathrm{m}$ below dura. a, Maximum intensity projection of a neuron in the mouse cortex (Thy1-YFP-H), at 863-875 $\mu \mathrm{m}$ below dura, under $1300 \mathrm{~nm}$ excitation, taken without and with AO. Post-objective power: $42 \mathrm{~mW}$. b, Zoomed-in views of the red box in a. $3 \times$ digital gain was applied to the image taken without $\mathrm{AO}$ to improve visibility. c, Signal profiles along the purple and blue lines in a. d, Corrective wavefront. Scale bar, $10 \mu \mathrm{m}$. Microscope objective: NA $1.0525 \times$.

Imaging beyond the mouse neocortex and into the hippocampus is difficult owing to the highly scattering white matter. Our AO module enabled imaging of hippocampal structures with subcellular resolution, without the need to perform any invasive procedure (e.g., cortical tissue removal, optical device insertion) other than the cranial window implantation (Supplementary Videos 5-8). Using an excitation wavelength of $1300 \mathrm{~nm}$, aberration correction significantly improved the 3P fluorescence signal ( 4-fold) of YFP-labeled somata (Fig. $2 \mathrm{e}$ and Supplementary Video 6), with many synaptic structures (e.g., dendritic spines) clearly resolvable only after AO correction (Fig. $2 f$ and Supplementary Video 7). In addition to 3P excitation of green fluorophores using $1300 \mathrm{~nm}$, excitation of red fluorophores with $1700 \mathrm{~nm}$ has been shown to improve tissue penetration ${ }^{1}$. To assess the performance of our module under $1700 \mathrm{~nm}$ excitation, we imaged tdTomato-labeled neuronal cell bodies at 952-1020 $\mu \mathrm{m}$ below dura, and found a similar improvement in the image signal and contrast after $\mathrm{AO}$ correction (Fig. 2i-k, Supplementary Fig. 9, and Supplementary Video 8).
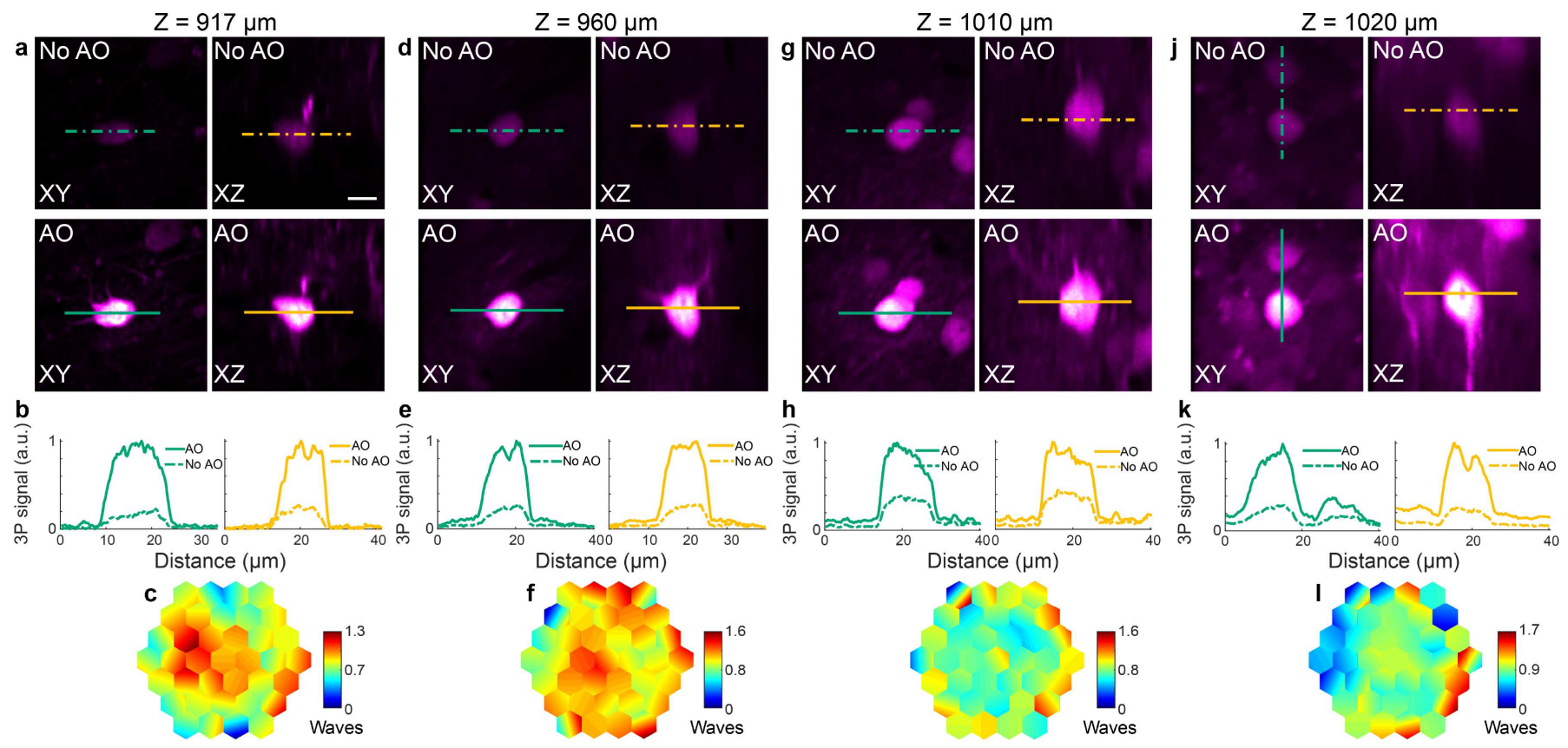

Supplementary Figure 9 | AO improves in vivo 3P imaging of hippocampal structures at different depths in the mouse brain, with $1700 \mathbf{~ n m}$ excitation. a,d,g,j, Lateral and axial images of neurons in the mouse hippocampus, without and with AO, at 917, 960, 1010, and $1020 \mu \mathrm{m}$ below dura, respectively. Post-objective powers: $26.5,10,27$, and $24 \mathrm{~mW}$, respectively. b,e,h,k, Signal profiles along the green and yellow lines in $\mathbf{a}, \mathbf{d}, \mathbf{g}$, and $\mathbf{j}$, respectively. c,f,i,l, Corrective wavefront in $\mathbf{a}, \mathbf{d}, \mathbf{g}$, and $\mathbf{j}$, respectively. For $\mathbf{a}$, a Gad2IRES-Cre $\times$ Ai14 (Rosa26-CAG-LSL-tdTomato) mouse was used; for $\mathbf{d}, \mathbf{g}$, and $\mathbf{j}$, neurons in wildtype mice were infected by a mix of AAV-Syn-Cre and AAV-CAG-FLEX-tdTomato. Scale bar, $10 \mu \mathrm{m}$. Microscope objective: NA 1.05 25×. 
The excellent imaging performance attained by incorporating our $\mathrm{AO}$ module into a $3 \mathrm{P}$ microscope motivated us to image neuronal structures in an even more challenging environment of the central nervous system: the spinal cord (Fig. 3a-g). The high neuronal density ${ }^{12}$, along with the surface curvature and strong scattering caused by superficial axon tracts, cause wavefront distortions which have prevented 2P microscopy from imaging neuronal structures beyond the most superficial $<200 \mu \mathrm{m}$ of the spinal cord dorsal horn ${ }^{13}-$ spanning a depth of roughly $500 \mu \mathrm{m}$. We performed in vivo 3P imaging of GFP-labeled neuronal structures at depths exceeding $400 \mu \mathrm{m}$ below dura through a dorsal laminectomy ${ }^{12}$, in 9- to 10-week old adult mice, under $1300 \mathrm{~nm}$ excitation (Fig. 3a-g). After aberration correction, we achieved signal improvements ranging from 2to 5 -fold (Fig. 3c,f) and more clearly visualized fine neuronal structures.

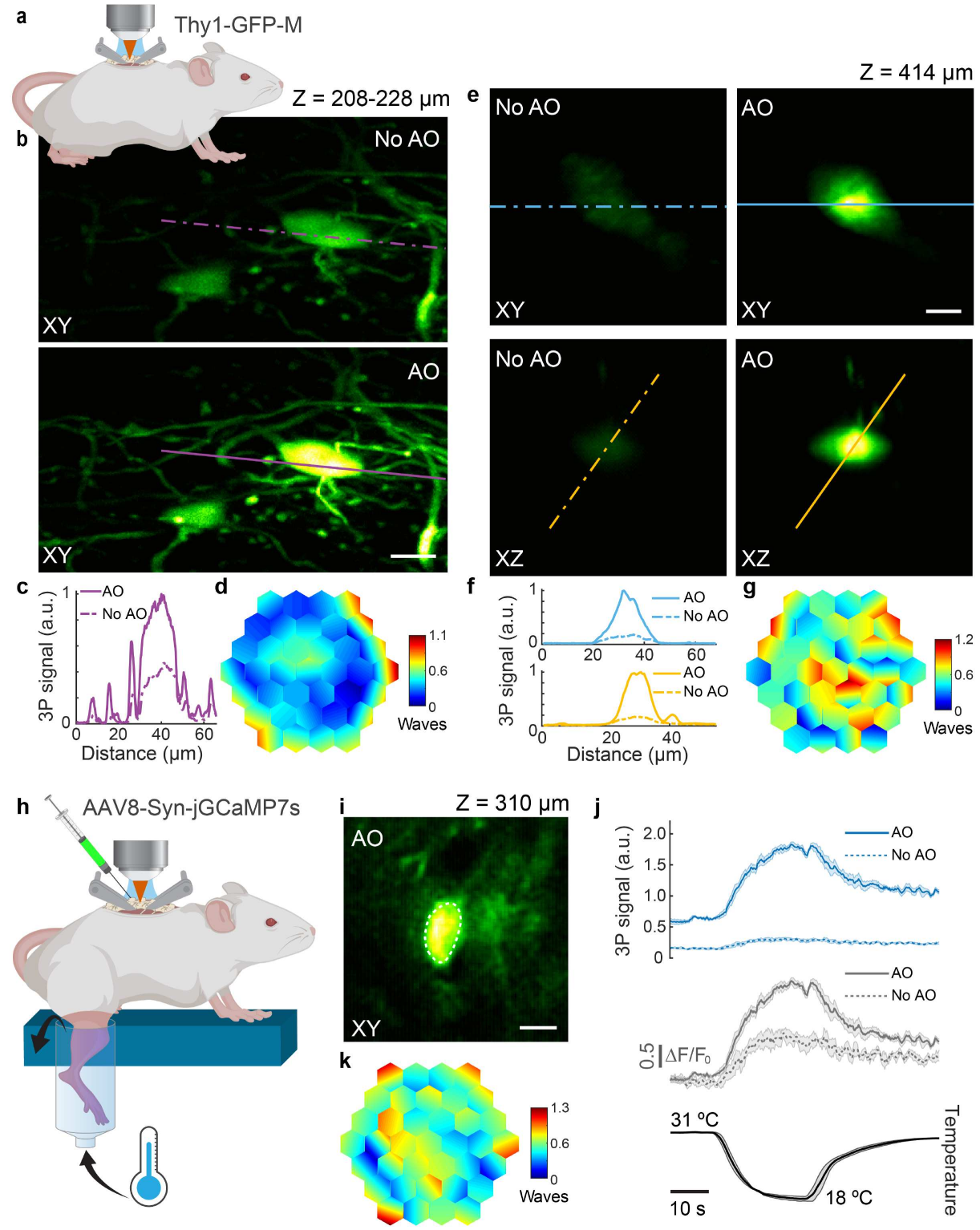

Fig. 3 AO improves in vivo 3P structural and functional imaging in the mouse spinal cord. a, Schematic of in vivo imaging in the dorsal horn of the mouse spinal cord. b, Maximum intensity projection of spinal cord neurons (Thy1-GFP-M), 208-228 $\mu \mathrm{m}$ below dura, under $1300 \mathrm{~nm}$ excitation, without and with AO (phase modulation). Post-objective power: $18.3 \mathrm{~mW}$. c, Signal profiles along the purple lines in b. d, Corrective wavefront in b. e, Lateral and axial images of a neuron (Thy1-GFP-M), $414 \mu \mathrm{m}$ below dura, under $1300 \mathrm{~nm}$ excitation, without and with AO (phase modulation). Post-objective power: $89 \mathrm{~mW}$. $\mathbf{f}$, Signal profiles along the blue and yellow lines in $\mathbf{e}$. $\mathbf{g}$, Corrective wavefront in $\mathbf{e}$. $\mathbf{h}$, Schematic for recording calcium activity in jGCaMP7s-expressing neurons of the dorsal horn in the mouse spinal cord (AAV8-Syn-jGCaMP7s), in response to cooling stimuli applied to the skin of the hindlimb. $\mathbf{i}$, Lateral image of a neuron, $310 \mu \mathrm{m}$ below dura, under $1300 \mathrm{~nm}$ excitation, after AO correction. j, (top) 3P fluorescence signal and (middle) calcium transients $\left(\triangle F / F_{0}\right)$, during (bottom) temperature stimulation, without and with $A O$ (phase modulation), for the neuronal cell body shown in i. 4-trial average; shaded area: s.e.m. Post-objective power: $4.2 \mathrm{~mW}$. k, Corrective wavefront in i. Scale bars, $10 \mu \mathrm{m}$. Microscope objective: NA $1.0525 \times$. 
Finally, our AO module enabled us to reliably record somatosensory-evoked calcium transients in the spinal cord dorsal horn at depths beyond $300 \mu \mathrm{m}$. Due to its optically challenging nature, such recordings had been limited to the most superficial $(<100 \mu \mathrm{m})$ layers, preventing a comprehensive understanding of the complex coding of somatosensory stimuli in the spinal cord circuitry. We recorded calcium transients in jGCaMP7s ${ }^{14}$-expressing spinal cord neurons, in response to cooling applied to the hindlimb skin, at imaging depths where temperature responses had not been previously studied (Fig. 3h-j and Supplementary Video $9)^{12}$. At an imaging depth of $310 \mu \mathrm{m}$ below dura, aberration correction substantially improved the signal of a neuronal cell body (by 5.9-fold) and increased the calcium transient amplitudes, with a 2.1-fold increase in the peak calcium-dependent fluorescence change $\left(\Delta F / F_{0}\right)(F i g .3 j)$. Although imaging depths exceeding $400 \mu m$ were possible, we did not find cooling-responding neurons at such depths.

To summarize, by combining a novel $\mathrm{AO}$ module into a 2P and 3P fluorescence microscope, we achieved drastic improvements in image quality, along with subcellular resolution, on a variety of biological structures in vivo at great depths. Owing to the higher order nonlinearity of 3P excitation, we observed a particularly large increase in 3P image signal and contrast from correcting tissue-induced aberrations. In the mouse brain, this combination allowed us to resolve synaptic structures in deep cortical layers as well as subcortical areas, otherwise invisible without $A O$ correction, while keeping post-objective average laser powers between $\approx 10-40$ $\mathrm{mW}$ (see Supplementary Table 1 for all imaging parameters). In the mouse spinal cord, our AO module enabled subcellular-resolution imaging of neuronal structures, at depths of more than twice of what previous studies had shown ${ }^{15}$. Moreover, aberration correction drastically improved the quality of somatosensoryevoked calcium transient recordings, at depths much beyond ( $>3 \times$ ) what had been previously reported ${ }^{12,16,17}$, opening the door for unravelling previously inaccessible spinal cord circuitry. Besides the ability to achieve subcellular resolution at depth, by improving the focus quality through aberration correction, the excitation power can be significantly reduced, minimizing the out-of-focus background (thus extending the imaging depth limit) and decreasing the power delivered to the sample well below the levels where heating-related effects ${ }^{18}$, or photo-damage ${ }^{19}$, would be a concern.

The high power throughput, ease of implementation, and small footprint needed by our AO module, along with its polarization- and wavelength-independent operation, provides easy integration into existing laserscanning multiphoton microscopes - such as 2P/3P microscopes, as well as other point-scanning modalities including those based on harmonic generation and Raman scattering. As such, our module can be adopted by a variety of biological laboratories, enabling the investigation of biological processes inside living tissues with subcellular resolution, in fields ranging from neurobiology and cancer biology to plant biology.

\section{Data availability}

The authors declare that the main data supporting the findings of this study are available within the paper and its supplementary information files. Additional data are available from the corresponding author on reasonable request.

\section{Acknowledgements}

We thank J. Wu for help with detection system; the Janelia JET team for designing and assembling the dispersion compensation unit; E. Carroll for help with galvo electronics; S. Chen for surgical assistance; R. Natan, K. Borges, and Q. Zhang for helpful discussions. This work was supported by the Howard Hughes Medical Institute (C.R., A.C., Y.L., W.S., D.E.M., and N.J.); the Burroughs Wellcome Fund under the Career Awards at the Scientific Interface (C.R.); Lawrence Berkeley National Laboratory LDRD 20-116 (J.A.R.); and the Firmenich Next Generation Fund, the Terman Fellowship, NIH grants R01DA045664, R01MH116904, and R01HL150566 (X.C.).

\section{Author contributions}

N.J. conceived and supervised the project. D.E.M., A.C., and N.J. developed the AO control program. T.G.B. performed the DM calibration and provided support on its operation. Y.L. and W.S. performed the mouse brain 
surgery. M.A.M. performed the mouse spinal cord surgery. M.A.M. built spinal cord temperature stimulation device and C.R. developed control program. A.C. built 2P AO setup and collected $2 \mathrm{P}$ data. C.R. built $3 \mathrm{P}$ AO setup and collected $3 P$ data in brain and beads. J.A.R. designed new $3 P$ system with input from C.R. J.A.R. and C.R. built new 3P system (used for spinal cord experiments). C.R. and M.A.M. designed and performed 3P imaging experiments in spinal cord. C.R. analyzed data and prepared all figures and supplementary material. X.K.C. supervised spinal cord experiments. C.R. and N.J. wrote the manuscript with feedback from M.A.M. and input from all other authors.

\section{Competing interests}

N.J. and Howard Hughes Medical Institute have filed patent applications that relate to the principle of frequency-multiplexed aberration measurement. T.G.B. has a financial interest in Boston Micromachines Corporation (BMC), which produced commercially the deformable mirror used in this work. 
Supplementary Table 1: Experimental parameters used for the aberration measurement data shown in this work

\begin{tabular}{|c|c|c|c|c|c|c|}
\hline Fig. & Imaging depth $(\mu \mathrm{m})$ & Post-objective power (mW) & Excitation wavelength $(\mathrm{nm})$ & AO iterations & Modulation strategy & mouse line/age/acute or chronic imaging \\
\hline $1 \mathrm{a}$ & 110 & 13 & 920 & 4 & phase & zebrafish line: $\operatorname{Tg}(\beta$-actin:HRAS-EGFP) \\
\hline $1 \mathrm{~d}$ & $365-375$ & 31 & 920 & 3 & phase & Thy1-YFP-H/>30wks/acute \\
\hline $1 \mathrm{~g}$ & $490-513$ & 128 & 920 & 3 & phase & Thy 1-YFP-H/>30wks/acute \\
\hline $2 \mathrm{a}$ & $747-767$ & 17 & 1300 & 3 & phase & Thy1-YFP-H/6wks/acute \\
\hline $2 \mathrm{~b}$ & $751-767$ & 20 & 1300 & 3 & phase & Thy1-YFP-H/6wks/acute \\
\hline $2 \mathrm{e}$ & 719 & 16 & 1300 & 3 & phase & Thy 1-YFP-H/>30wks/chronic \\
\hline $2 f$ & $695-709$ & 26 & 1300 & 3 & phase & Thy1-YFP-H/>30wks/chronic \\
\hline $2 \mathrm{i}$ & 952 & 30 & 1700 & 3 & phase & Gad2-IRES-Cre Jax X Ai14/>30wks/chronic \\
\hline $3 \mathrm{~b}$ & $208-228$ & 18.3 & 1300 & 2 & phase & Thy1-GFP-M/10wks/acute \\
\hline 3 e & 414 & 89 & 1300 & 3 & phase & Thy1-GFP-M/9wks/acute \\
\hline $3 i$ & 310 & 4.2 & 1300 & 2 & phase & AAV8-Syn-jGCaMP7s/5wks/acute \\
\hline Supp. Fig. & Imaging depth $(\mu \mathrm{m})$ & Post-objective power (mW) & Excitation wavelength $(\mathrm{nm})$ & AO rounds & Modulation strategy & mouse line/age/acute or chronic imaging \\
\hline $1 \mathrm{c}$ & $\mathrm{N} / \mathrm{A}$ & 0.13 & 1300 & 1 & phase & $\mathrm{N} / \mathrm{A}$ \\
\hline $2 \mathrm{c}$ & N/A & 6 & 920 & 3 & phase & $\mathrm{N} / \mathrm{A}$ \\
\hline $2 d$ & N/A & 2.3 & 920 & 4 & phase & N/A \\
\hline $3 \mathrm{~b}$ & $\mathrm{~N} / \mathrm{A}$ & 0.13 & 1300 & 4 & phase & $\mathrm{N} / \mathrm{A}$ \\
\hline $4 a$ & 757 & 17 & 1300 & 3 & phase & Thy1-YFP-H/6wks/acute \\
\hline 4 e & $747-757$ & 20 & 1300 & 3 & phase & Thy1-YFP-H/6wks/acute \\
\hline 5 a & 623 & 20.8 & 1300 & 5 & phase & Thy 1-YFP-H/>30wks/chronic \\
\hline $5 \mathrm{~d}$ & 623 & 23.6 & 1300 & 5 & intensity & Thy 1-YFP-H/>30wks/chronic \\
\hline 6 a & $601-616$ & 17 & 1300 & 4 & phase & Thy 1-YFP-H/>30wks/chronic \\
\hline $6 \mathrm{~d}$ & $609-619$ & 25.6 & 1300 & 4 & phase & Thy 1-YFP-H/>30wks/chronic \\
\hline 7 a & 687 & 35 & 1300 & 2 & phase & Thy1-GFP-M/>30wks/acute \\
\hline $7 \mathrm{~d}$ & $624-644$ & 13 & 1300 & 2 & phase & Thy1-GFP-M/5wks/acute \\
\hline 8 a & $863-875$ & 42 & 1300 & 2 & phase & Thy1-YFP-H/5wks/acute \\
\hline $9 \mathrm{a}$ & 917 & 26.5 & 1700 & 3 & phase & Gad2-IRES-Cre $\times$ Ai14/>30wks/chronic \\
\hline $9 \mathrm{~d}$ & 960 & 10 & 1700 & 3 & phase & AAV-Syn-Cre + AAV-CAG-FLEX-tdTomato/11wks/acute \\
\hline $9 \mathrm{~g}$ & 1010 & 27 & 1700 & 3 & phase & AAV-Syn-Cre + AAV-CAG-FLEX-tdTomato/11wks/acute \\
\hline $9 \mathrm{j}$ & 1020 & 24 & 1700 & 3 & phase & AAV-Syn-Cre + AAV-CAG-FLEX-tdTomato/11wks/acute \\
\hline 10 & \multicolumn{6}{|c|}{ N/A } \\
\hline 11 & \multicolumn{6}{|c|}{$\mathrm{N} / \mathrm{A}$} \\
\hline 12 & $\mathrm{~N} / \mathrm{A}$ & 2 & 1300 & N/A & N/A & Thy1-YFP-H/6wks/acute \\
\hline
\end{tabular}




\section{References}

1. Horton, N. G. et al. In vivo three-photon microscopy of subcortical structures within an intact mouse brain. Nat. Photonics 7, 205-209 (2013).

2. Ouzounov, D. G. et al. In vivo three-photon imaging of activity of GcamP6-labeled neurons deep in intact mouse brain. Nat. Methods 14, 388-390 (2017).

3. Wang, T. \& Xu, C. Three-photon neuronal imaging in deep mouse brain. Optica 7, 947-960 (2020).

4. Joel A Kubby. Adaptive Optics for Biological Imaging. (CRC Press, 2013).

5. Booth, M. J. Adaptive optical microscopy: The ongoing quest for a perfect image. Light Sci. Appl. 3, 1-7 (2014).

6. Ji, N. Adaptive optical fluorescence microscopy. Nat. Methods 14, 374-380 (2017).

7. Rodríguez, C. \& Ji, N. Adaptive optical microscopy for neurobiology. Curr. Opin. Neurobiol. 50, 83-91 (2018).

8. Wang, K. et al. Direct wavefront sensing for high-resolution in vivo imaging in scattering tissue. Nat. Commun. 6 , 1-6 (2015).

9. Liu, R., Li, Z., Marvin, J. S. \& Kleinfeld, D. Direct wavefront sensing enables functional imaging of infragranular axons and spines. Nat. Methods 16, 615-618 (2019).

10. Wang, C. et al. Multiplexed aberration measurement for deep tissue imaging in vivo. Nat. Methods 11, 1037-1040 (2014).

11. Liu, R., Milkie, D. E., Kerlin, A., MacLennan, B. \& Ji, N. Direct phase measurement in zonal wavefront reconstruction using multidither coherent optical adaptive technique. Opt. Express 22, 1619-1628 (2014).

12. Ran, C., Hoon, M. A. \& Chen, X. The coding of cutaneous temperature in the spinal cord. Nat. Neurosci. 19, 12011209 (2016).

13. Cheng, Y.-T., Lett, K. M. \& Schaffer, C. B. Surgical preparations, labeling strategies, and optical techniques for cell-resolved, in vivo imaging in the mouse spinal cord. Exp. Neurol. 318, 192-204 (2019).

14. Dana, H. et al. High-performance calcium sensors for imaging activity in neuronal populations and microcompartments. Nat. Methods 16, 649-657 (2019).

15. Matsumura, S., Taniguchi, W., Nishida, K., Nakatsuka, T. \& Ito, S. In vivo two-photon imaging of structural dynamics in the spinal dorsal horn in an inflammatory pain model. Eur. J. Neurosci. 41, 989-997 (2015).

16. Johannssen, H. C. \& Helmchen, F. In vivo $\mathrm{Ca}^{2+}$ imaging of dorsal horn neuronal populations in mouse spinal cord. J. Physiol. 588, 3397-3402 (2010).

17. Sekiguchi, K. J. et al. Imaging large-scale cellular activity in spinal cord of freely behaving mice. Nat. Commun. 7 , 11450 (2016).

18. Podgorski, K. \& Ranganathan, G. Brain heating induced by near-infrared lasers during multiphoton microscopy. J. Neurophysiol. 116, 1012-1023 (2016).

19. Olivié, G. et al. Wavelength dependence of femtosecond laser ablation threshold of corneal stroma. Opt. Express 16, 4121-4129 (2008).

20. Akturk, S., Gu, X., Kimmel, M. \& Trebino, R. Extremely simple single-prism ultrashort- pulse compressor. Opt. Express 14, 10101-10108 (2006).

21. Horton, N. G. \& Xu, C. Dispersion compensation in three-photon fluorescence microscopy at 1,700 nm. Biomed. Opt. Express 6, 1392-1397 (2015).

22. Stewart, J. B. et al. Design and development of a 331-segment tip-tilt-piston mirror array for space-based adaptive optics. Sensors Actuators, A Phys. 138, 230-238 (2007).

23. Stewart, J. B., Diouf, A., Zhou, Y. \& Bifano, T. G. Open-loop control of a MEMS deformable mirror for largeamplitude wavefront control. J. Opt. Soc. Am. A 24, 3827 (2007).

24. Ji, N., Milkie, D. E. \& Betzig, E. Adaptive optics via pupil segmentation for high-resolution imaging in biological tissues. Nat. Methods 7, 141-147 (2010).

25. Bridges, W. B. et al. Coherent Optical Adaptive Techniques. Appl. Opt. 13, 291-300 (1974).

26. O'Meara, T. R. Theory of multidither adaptive optical systems operating with zonal control of deformable mirrors. J. Opt. Soc. Am. 67, 318-325 (1977).

27. O’Meara, T. R. The multidither principle in adaptive optics. J. Opt. Soc. Am. 67, 306-315 (1977). 
28. Turcotte, R., Liang, Y. \& Ji, N. Adaptive optical versus spherical aberration corrections for in vivo brain imaging. Biomed. Opt. Express 8, 3891-3902 (2017).

29. Thévenaz, P., Ruttimann, U. E. \& Unser, M. A pyramid approach to subpixel registration based on intensity. IEEE Trans. Image Process. 7, 27-41 (1998).

30. Godinho, L. Imaging zebrafish development. Cold Spring Harb. Protoc. 2011, 879-83 (2011).

31. Sun, W., Tan, Z., Mensh, B. D. \& Ji, N. Thalamus provides layer 4 of primary visual cortex with orientation- and direction-tuned inputs. Nat. Neurosci. 19, 308-315 (2016).

\section{Methods}

Animals. All animal experiments were conducted according to the National Institutes of Health guidelines for animal research. Procedures and protocols on mice and zebrafish were approved by the Institutional Animal Care and Use Committee at Janelia Research Campus, Howard Hughes Medical Institute; and the Animal Care and Use Committee at the University of California, Berkeley. Details on animal preparations are available below.

Excitation Source. The 2P excitation source was a mode-locked titanium:sapphire laser (Chameleon Ultra II; Coherent) operating at $920 \mathrm{~nm}$. The 3P excitation source consisted of a two-stage optical parametric amplifier (Opera-F; Coherent) pumped by a $40 \mathrm{~W}$ diode-pumped femtosecond laser (Monaco 1035-40-40; Coherent) operating at $1035 \mathrm{~nm}$ and $1 \mathrm{MHz}$, providing a broad tuning range (650-920 nm and 1200-2500 nm). Opera-F was operated at 1300 and $1700 \mathrm{~nm}$ for $3 \mathrm{P}$ excitation, for which the average output power was $\sim 1.5$ and $\sim 0.9$ $\mathrm{W}(1.5$ and $0.9 \mu \mathrm{J}$ per pulse at $1 \mathrm{MHz}$ repetition rate), respectively. For $1300 \mathrm{~nm}$ excitation, to reduce the group delay dispersion (GDD) at the sample plane, we used a homebuilt single-prism compressor ${ }^{20}$. After compensation, the pulse duration at the focal plane of the objective was measured to be $\sim 54 \mathrm{fs}$ using an autocorrelator (Carpe, APE GmbH). For $1700 \mathrm{~nm}$ excitation, since the GDD is anomalous for many of the glasses and crystals used in our microscope, the resulting negative GDD at the sample plane cannot be compensated for using our prism-based compressor. Instead, the high normal dispersion of ZnSe (from a bulk compressor available inside Opera-F) and silicon (from a 3-mm thick window placed at Brewster's angle ${ }^{21}$ ) was used to obtain a pulse duration at the sample of $\sim 70 \mathrm{fs}$ after compensation.

Adaptive optical microscope setup. Simplified diagrams of our homebuilt $2 \mathrm{P}$ microscope and $3 \mathrm{P}$ microscope are shown in Supplementary Fig. 1.

For the 2P microscope (Supplementary Fig. 1a), a pair of achromat doublets (AC254-200-B and AC254300-B; Thorlabs) conjugated the segmented deformable mirror (DM) surface to a liquid-crystal spatial light modulator (SLM; Holoeye, PLUTO-NIR) used to introduce artificial aberrations. The SLM plane was then conjugated to a pair of galvanometers $(6215 \mathrm{H}$; Cambridge Technology) that were optically conjugated to each other and the back focal plane of a high-numerical aperture (NA) water-dipping objective (Olympus XLPLN25XWMP2, NA 1.05, 25x; or Nikon CFI LWD, NA 0.8, 16×), using three pairs of achromat doublets (AC254-150-B and AC254-60-B, AC508-080-B and AC508-080-B, AC508-75-C and SLB-50-600PIR1; Thorlabs and OptoSigma).

For the 3P microscope, femtosecond pulses at 1300 or $1700 \mathrm{~nm}$ were reflected off a segmented deformable mirror (DM). The DM was conjugated to a pair of galvanometers $(6215 \mathrm{H}$; Cambridge Technology) that were optically conjugate to each other and the back focal plane of a high- NA water-dipping objective (Olympus XLPLN25XWMP2, NA 1.05, 25x), using three pairs of achromat doublets (Original 3P system: AC254-100-C and 45-804, AC508-080-C and AC508-080-C, AC508-100-C and SLB-50-600PIR2; Thorlabs, Edmund Optics, and OptoSigma. New 3P system: AC254-400-C and AC254-300-C, SL50-3P and SL50-3P, SL50-3P and TTL200MP; Thorlabs).

For both the $2 \mathrm{P}$ and $3 \mathrm{P}$ microscopes, a field stop (iris diaphragm, Thorlabs) was located at the intermediate image plane between the DM and the $\mathrm{X}$ galvo to block unwanted diffraction orders and light reflected off mirror segments at large tilt angles. To translate the focus axially, the objective was mounted on a piezoelectric stage 
(P-725.4CD PIFOC; Physik Instrumente). The fluorescence signal was collected by the same objective and reflected from a dichroic beam splitter (FF665-Di02-25x36; Semrock), spectrally filtered (2P: FF01-680/SP, Semrock. 3P: FF01-680/SP, Semrock; together with BLP01-442R-25, Semrock, or ET575Ip, Chroma, to block the third-harmonic generation signal at 1300 and $1700 \mathrm{~nm}$, respectively), and detected by a photomultiplier tube (H7422-40 or H10770PA-40; Hamamatsu). A Pockels cell was used for controlling the excitation power (2P: M350-80; 3P: M360-40; Conoptics). For the experiments using $1700 \mathrm{~nm}$ excitation, we used $\mathrm{D}_{2} \mathrm{O}$, instead of $\mathrm{H}_{2} \mathrm{O}$, for the objective immersion media, because of its much lower absorption ${ }^{21}$. Custom-written software was used for image acquisition.

Deformable mirror (DM) specifications, calibration, and verification procedure. The deformable mirror in this project is a hexagonal tip-tilt-piston DM (Hex-111-X) manufactured by Boston Micromachines Corporation (Supplementary Fig. 10). The device features 37 hexagonal segments, each anchored to three electrostatic actuators by short attachment posts, a 3.8-mm-diameter clear aperture, and a protective window with antireflection coating (400 nm to $1100 \mathrm{~nm}$ for $2 \mathrm{P}$ experiments and $550 \mathrm{~nm}$ to $2400 \mathrm{~nm}$ for $3 \mathrm{P}$ experiments). We developed it for microscopy application from a 331-segment tip-tilt-piston mirror array originally designed for space-based $\mathrm{AO}^{22,23}$. The actuators are independently addressable and can achieve a maximum of $3.5 \mu \mathrm{m}$ of surface-normal stroke for an input voltage of $\sim 200 \mathrm{~V}$. Tip, tilt, and piston control of mirror segments is achieved by applying command voltages to each of the three actuators. The electromechanical system has no measurable hysteresis. The maximal segment piston motion achievable is $3.5 \mu \mathrm{m}$, and the maximal tip and tilt angle achievable is $\pm 5.7 \mathrm{mrad}$. However, these maxima are not independent. The range of tip and tilt angle achievable depends on the nominal piston value, and vice versa. Coupling forces and torques are generated on the actuators through their mechanical connection to the mirror segment. As a result, the surface normal deflection at a given actuator post depends not only on the voltage applied to that actuator, but also on the state of its neighboring actuators. Nevertheless, there is a one-to-one mapping between the voltages applied to the three actuators and the resulting tip, tilt, and piston orientations of the segment.

a

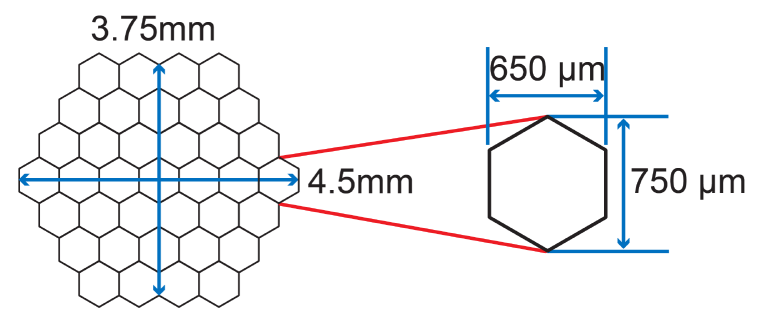

b

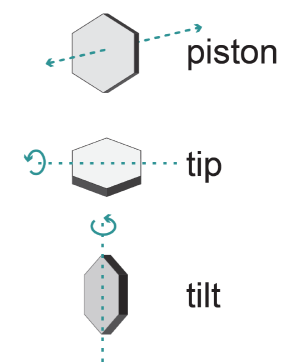

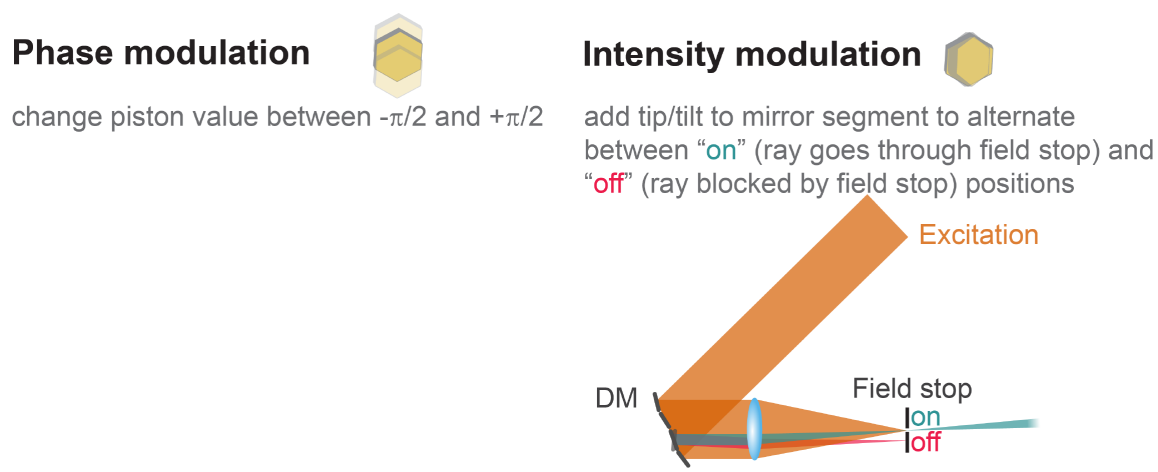

Supplementary Figure 10 | Deformable mirror (DM) specifications. a, Diagram of the hexagonal segmented DM (Boston Micromachines Corporation) consisting of 37 segments and a 3.8-mm-diameter clear aperture. $\mathbf{b}$, Each segment is controlled by three actuators to provide an angular control of approximately \pm 3 mrad in tip and tilt, and a maximum axial stroke (piston displacement) of $3.5 \mu \mathrm{m}$. c, Diagram showing the two modulation strategies used by our aberration measurement method. See Online Methods for details.

A calibration process was used to fit a mathematical model to that mapping. During calibration, the tip, tilt, and piston of each segment was measured to nanometer-scale precision using a surface mapping 
interferometer (Zygo 6100) in response to each of 2744 input voltage states applied to the actuators (with voltages ranging from $0 \mathrm{~V}$ to $200 \mathrm{~V}$ in 14 steps for each actuator). A least squares fourth order polynomial fit was made between the input state (the vector comprised of the three actuator input voltages) and the output state (the vector comprised of the three segment orientations, tip, tilt, and piston). The correlation coefficient of the fit was 1.000. This fit was used to create a lookup table that could be interpolated to map any desired output orientation to a corresponding triplet of required actuator input voltages. The calibration was tested in the interferometer, and yielded precision corresponding to approximately $1 \mathrm{~nm}$ in piston motion and 0.1 uradians in tip and tilt.

The calibration of the DM was further tested in our microscope setup. Piston displacement was verified by recording the fluorescence signal from a 1- $\mu \mathrm{m}$ diameter fluorescent bead while piston displacing one mirror segment. A sinusoidal signal trace with constant amplitude and period equal to $\lambda / 4$ ( $\lambda$, excitation wavelength) confirmed an accurate calibration and proper functioning of the mirror segment. The same was repeated for all DM segments. Tip/tilt displacement was verified by recording the image of a $10-\mu \mathrm{m}$ diameter fluorescent bead while scanning a single DM segment along the maximum tip/tilt range. The light reflected off the remaining segments was blocked by the field stop. Using a custom-written MATLAB code, the centroid of the bead was determined for each tip/tilt position, and a plot of measured vs. expected tip/tilt was calculated, taking into consideration the magnification factors in our microscope setup. A slope approximately equal to 1 confirmed accurate calibration of the mirror segment. The same was repeated for all remaining segments. The settling times of the mirror segments were found to be $<100 \mu \mathrm{s}$, which allowed us to carry out kHz modulation of the light impinging on it.

Aberration measurement method. The general aberration measurement procedure was conceptually similar to that described in our previous work ${ }^{10}$. Experimentally, the laser focus was parked at one sample location and the fluorescence signal from this location was used for aberration measurement. The pupil was segmented to 37 regions, corresponding to the number of segments in the DM. The 37 segments were separated into two groups of alternating rows (Supplementary Fig. 11).

First, we fixed the tip, tilt, and piston of one group of 17 segments, but added to each of the other 20 pupil segments a specific tip angle $\Theta_{i}$ and tilt angle $\Phi_{j}(i, j=1,2, \ldots n)$ (Supplementary Fig. 11), Step 1), each of which were chosen randomly from an array of $n$ angles evenly spaced between $-\Psi / 2$ and $\Psi / 2$. These applied tip/tilt angles caused the beamlet reflecting off the segment to displace along the $X$ and $Y$ axes in the objective focal plane by $X_{i}$ and $Y_{j}\left(X_{i}=f^{\star} \tan \left(2 \Theta_{i} / M\right)\right.$ and $Y_{j}=f^{\star} \tan \left(2 \Phi_{j} / M\right)$; $f$ : focal length of the objective; $M$ : magnification from the DM to objective back focal plane), respectively, which changed their interference with the reference focus formed by the other 17 beamlets. With the tip and tilt angles of all segments fixed, using the segments themselves (see details in "Modulation strategies") we then modulated the phase or intensity of all 20 beamlets, each at a distinct frequency $\omega_{s}(s=1,2, \ldots 20)$, and recorded the fluorescence signal for a time duration T (Supplementary Fig. 11, Step 2). The recorded signal trace was then Fourier transformed (FT) and the Fourier magnitudes at each distinct modulation frequency $\omega_{s}$ were measured (Supplementary Fig. 11, Step $3)$, whose values indicated how much individual beamlets interfered with the reference focus at the focal displacement of $\left(X_{i}, Y_{j}\right)$.

The above procedure was repeated $n \times n$ times, so that all 20 segments sampled the full tip/tilt angles and their corresponding beamlets scanned over a 2D grid in the focal plane with the dimensions $2 f^{\star} \tan (\Psi / M)$ by $2 f^{\star} \tan (\Psi / M)$ (Supplementary Fig. 11, Step 4). For each beamlet, plotting the Fourier magnitudes versus the displacements $\left(X_{i}, Y_{j}\right)$, we constructed a 2D map of interference strength of this beamlet with the reference focus at different focal displacements (Supplementary Fig. 11, Step 5). Fitting the map with a 2D Gaussian function, we found the displacements leading to maximal interference between the beamlets and their reference focus, which gave us the tip/tilt angles (i.e., phase gradient) to be applied to this segment in the corrective wavefront.

We then repeated Steps 1-5, but now with the group of 20 pupil segments fixed and forming the reference focus. Modulating the remaining 17 segments, we obtained their $2 \mathrm{D}$ maps of interference strength versus displacement (Supplementary Fig. 11, Step 6) and the phase gradients required to shift the corresponding beamlets to coincide with the reference focus. The total fluorescence signal acquisition time was $2 \times n \times n \times \mathrm{T}$. 
With all the beamlets intersecting at the same location, the next step was to determine the phase offsets that would allow them to constructively interfere ${ }^{24}$. Consider the case of finding the phase offset that enables two beamlets to constructively interfere. With one beamlet assigned as the reference (with unknown phase $\theta_{r}$ ), by incrementally adding a phase offset $\Delta \theta$ to the other beamlet (with unknown phase $\theta_{1}$ ) at step size $\omega$ (i.e., $\Delta \theta=\omega \mathrm{t})$, the intensity variation can be described by $I=2+2 \cos \left(\omega \mathrm{t}+\theta_{1}-\theta_{r}\right)$. The phase offset that gives the maximal intensity (i.e., constructive interference) is thus the opposite of the phase of the function cos $(\omega t+$ $\left.\theta_{1}-\theta_{r}\right)$. One approach is by Fourier-transforming the time-dependent signal and reading out the phase at frequency $\omega / 2 \pi$. To determine the phase offsets of 37 segments, we employed the concept of multidither coherent optical adaptive technique ${ }^{11,25-27}$. We modulated the phases of the first 20 rays by piston-displacing each corresponding mirror segment at a distinct frequency $\omega_{s}$ while keeping the phases of the remaining rays, which formed a reference focus, constant. We then Fourier transformed the recorded fluorescence trace (Supplementary Fig. 11, Step 7), and read out the phase offsets that would lead to constructive interference with the reference focus at the modulation frequencies $\omega_{s}(s=1,2, \ldots 20)$ (Supplementary Fig. 11, Step 8). We next modulated the phases of the remaining 17 segments while keeping the phases of the first 20 segments unchanged and found the phase offsets for these 17 segments required to ensure constructive interference among beamlets.

With all beamlets intersecting and constructively interfering at the focal plane, we obtained the final corrective wavefront and applied it to the DM for aberration correction. Because the reference foci used for both phase gradient measurements (Supplementary Fig. 11, Step 1-6) and phase offset measurements (Supplementary Fig. 11, Step 7-9) were aberrated to begin with, for larger aberrations, the whole procedure was iterated, as needed, to achieve optimal aberration correction (Supplementary Fig. 11, Step 10). 


\section{Phase gradient measurement}

(1) Apply tip/tilt to first group of segments

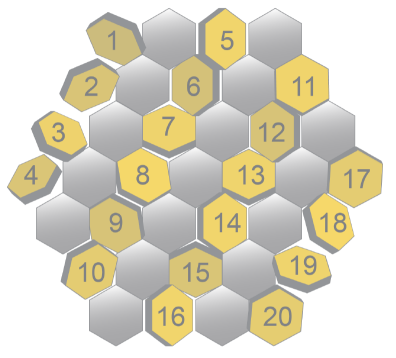

(2) Modulate each segment at a distinct frequency $\omega_{s}$ \& measure signal during modulation

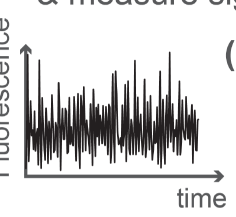

(3) Fourier-transform the signal trace and read the FT magnitudes at the modulation frequencies in (2) (orange data points)

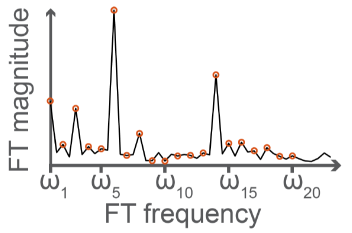

(4) Repeat $n \times n$ times for all possible tip/tilt combinations and obtain the corresponding FT magnitudes

(5) Plot the FT magnitude for each tip/tilt combination to create a 2D interference-vs-displacement map

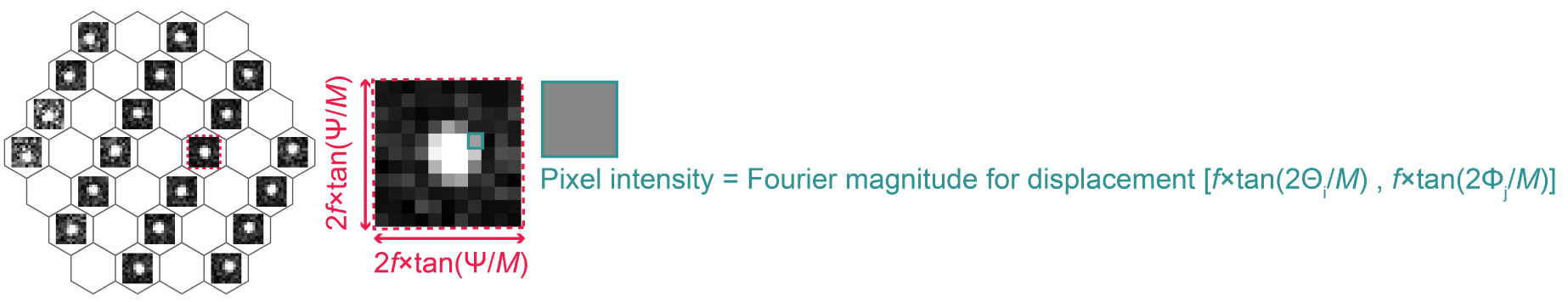

(6) Repeat (1)-(5) for the second group of segments

\section{ala 10}

\section{adon}

101010 Fit each map with a 2D Gaussian function and find the displacements leading to maximal

101010 interference between the light ray and the reference focus, which correspond to the tip/tilt angles

A1010 (i.e., phase gradient) for each segment of the corrective wavefront

Diala

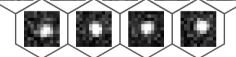

\section{Phase offset measurement}

(7) Modulate the phase of first group of segments, each at a distinct frequency $\omega_{s}$, and measure signal during modulation

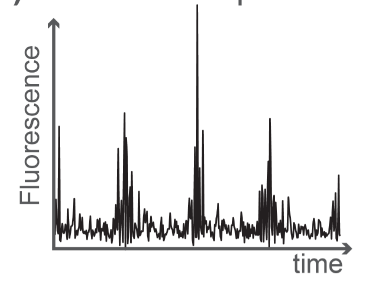

(8) Fourier-transform the signal trace and read the phases at the modulation frequencies in (7) (orange data points)

(9) Repeat (7)-(8) for the secong group of segments and obtain the corrective wavefront

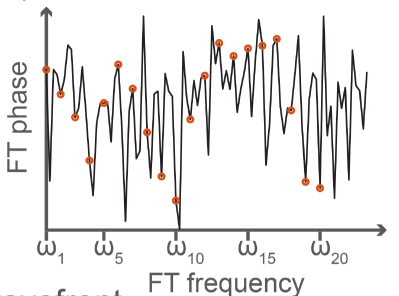

(10) Repeat the steps above as needed to obtain the final corrective wavefront and apply it to the DM

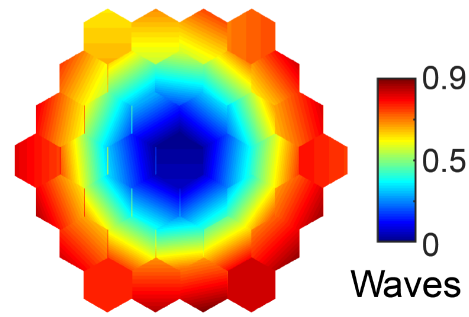

Final corrective wavefront

Supplementary Figure 11 | Schematics of the aberration measurement method. See detailed description in Methods. 
Modulation strategies. Two different modulation strategies were used: phase modulation and intensity modulation. During phase modulation, mirror segments were piston-displaced between - $\pi / 2$ and $\pi / 2$ (imparting the beamlets reflecting off them a phase shift ranging from $-\pi$ to $\pi$ ) at frequency $\omega_{s}$, resulting in a modulation on the detected fluorescence signal. During intensity modulation, a large tip (or tilt) angle was intermittently applied to the mirror segments being modulated, each at a unique $\omega_{s}$, such that the beamlets impinging on them alternate between two positions: the "on" position, where beamlets went through the field stop placed after the DM; and the "off" position, where beamlets were blocked by the field stop (Supplementary Fig. 10).

During phase modulation, the modulation in the fluorescence signal originates from the varying phase offsets between the modulated rays and the reference focus. In contrast, during intensity modulation, the periodic variation in the fluorescence signal comes from the changes in excitation power at the focal plane. While for phase modulation there is always a maximum present in $2 \mathrm{D}$ maps of interference strength versus displacement, for intensity modulation, if the relative phase between the ray and the reference focus is near $\pi / 2$, a clear maximum would be absent from the resulting map, in which case a $\pi / 2$ phase offset needs to be added to the corresponding segment and the measurement redone.

Depending on the sample, fluorescent features of different sizes may be used for aberration measurement. We systematically tested and compared the performance of phase versus intensity modulation, each of which was used to measure artificial aberrations using the signal from fluorescent features of different sizes (Supplementary Fig. 2). Experimentally we found that, for $2 \mathrm{P}$ microscopy, phase modulation outperformed intensity modulation when signal from small features $(<4 \mu \mathrm{m})$ was used for aberration measurement, whereas intensity modulation led to faster improvement of image quality for features larger than $4 \mu \mathrm{m}$. For $3 \mathrm{P}$ microscopy, phase modulation was found to perform better than or equally with intensity modulation for all feature sizes tested (from 1- $\mu \mathrm{m}$ diameter fluorescent beads to a fluorescent solution). Similar performance between phase and intensity modulation was validated in vivo by performing the correction using a neuronal cell body (Supplementary Fig. 7).

Typical operation parameters and imaging considerations. An example set of operation parameters used for aberration measurement in the mouse brain in vivo (Fig. 2) included an integration time of $\mathrm{T}=90 \mathrm{~ms}$ for each of the $11 \times 11(n \times n)$ tip/tilt angles, which scanned the modulated rays over $19 \mu \mathrm{m} \times 19 \mu \mathrm{m} 2 \mathrm{D}$ grids in the focal plane. The overall fluorescence acquisition time was therefore $21.8 \mathrm{~s}(2 \times n \times n \times \mathrm{T})$. Additionally, for the phase measurement portion of the algorithm, the total fluorescence acquisition time was $1.8 \mathrm{~s}(360 \mathrm{~ms}$ per iteration, with a total of 5 iterations). Additional hardware (e.g., DM settling time) and software overheads added to the fluorescence acquisition time and the overall time used for aberration measurement and correction was $3-4 \times$ the fluorescence acquisition time. For data in Fig. 2, 3 rounds of aberration measurements were performed to obtain the final corrective wavefront.

"No AO" images were taken after system aberration correction (Supplementary Fig. 1c-e), as well as the adjustment of the objective correction collar to compensate for the spherical aberration introduced by the glass windows overlaying the mouse brain and spinal cord. Furthermore, to minimize additional aberration modes that arose from a tilted window and sample (e.g., coma ${ }^{28}$, we used the third-harmonic generation signal from the window-tissue interface to determine the angle of the window (Supplementary Fig. 12) and adjusted the mouse using a 2D goniometer stage till the window was perpendicular to the excitation beam. These procedures constitute the best practice and lead to the best performance that conventional optics could achieve. The deterioration in image signal and contrast observed in our "No AO" images, therefore, resulted from tissue-induced aberrations exclusively, and could only be compensated by AO. 


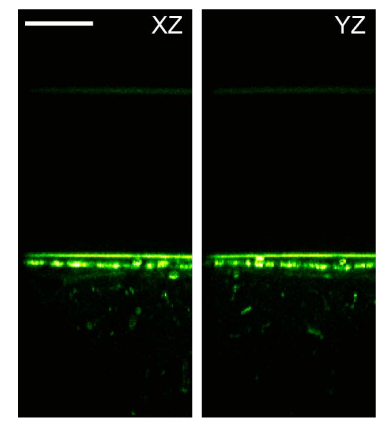

Supplementary Figure 12 | Cranial window alignment using the third-harmonic signal generated at the glass window - brain interface. Axial third-harmonic generation images of a cranial window above a Thy1-YFP-H mouse brain in vivo. Both the $3 \mathrm{P}$ fluorescence and the third-harmonic signals are shown, where the third-harmonic signal generated at the interfaces of the coverglass are clearly distinguished. Excitation wavelength: $1300 \mathrm{~nm}$ (same imaging session as in Fig. 2 a-d). Post-objective power: 2 mW. Scale bar, $50 \mu \mathrm{m}$. Microscope objective: NA $1.0525 \times$.

Digital image processing. Due to brain motion at depth, the StackReg ${ }^{29}$ image registration plug-in in ImageJ was used for rigid registration in 2D. The "smooth" function from ImageJ that replaced the value of each pixel with the average of $3 \times 3$ pixels centering on this pixel was applied to all $3 \mathrm{P}$ images. $2 \mathrm{P}$ images were presented using the "Green hot" lookup table in ImageJ; 3P images were presented with the "Green hot" and "Magenta hot" lookup tables for 1300 and $1700 \mathrm{~nm}$ excitation, respectively. For images where the signal was too weak before AO correction, a linear scaling factor was applied to all pixel values to improve visibility with the scaling factor listed on the image. The images presented did not undergo any other digital manipulation.

Bead samples. Carboxylate-modified fluorescent microspheres (Fluosphere ${ }^{\text {TM}}$; Invitrogen) were immobilized on poly(I-lysine)-coated microscope slides (12-550-12, Fisher Scientific).

Zebrafish preparation. Zebrafish procedures have been described previously ${ }^{30}$. Briefly, $\operatorname{Tg}(\beta$-actin:HRASEGFP) zebrafish embryos (Danio rerio) were grown at $28{ }^{\circ} \mathrm{C}$ in E3 zebrafish embryo medium. To generate optically transparent embryos, melanin synthesis was inhibited by transferring the embryos into $1 \times$ phenylthiourea (PTU) solution in E3 medium, 10-16 hours post fertilization. Before imaging, the chorions were manually removed with forceps under a stereomicroscope. Four-day-old larvae were then anesthetized in E3 medium containing $1 \times$ tricaine and immobilized on a dish by embedding them in $0.5 \%$ low-melting point agarose with $1 \times$ PTU and $1 \times$ tricaine. During imaging, E3 medium containing $1 \times$ PTU and $1 \times$ tricaine was used as immersion medium.

Mouse preparation (brain imaging). Cranial window implantation procedures were performed ${ }^{31}$, using aseptic technique, on mice that were anaesthetized with isoflurane (1-2\% by volume in 02 ) and given the analgesic buprenorphine (SC, $0.3 \mathrm{mg}$ per kg of body weight). For 2P imaging experiments, a 3.5-mm diameter craniotomy was made over V1 with dura left intact. A glass window made of two coverslips (Fisher Scientific, thickness no. 1.5) bonded with ultraviolet cured optical adhesives (Norland Optical Adhesives 61) was embedded in the craniotomy and sealed in place with dental acrylic. For 3P imaging experiments, a 5-mm diameter craniotomy was made over V1, with dura left intact. The glass window consisted of a donut-shaped coverslip (inner diameter $4.5 \mathrm{~mm}$, outer diameter $5.5 \mathrm{~mm}$; Potomac Photonics) bonded with ultraviolet cured optical adhesives (Norland Optical Adhesives 61) on top of a 5-mm diameter coverslip (Denville Scientific, thickness no. 1). The window was embedded in the craniotomy and sealed in place with dental acrylic. A titanium head-post was attached to the skull with cyanoacrylate glue and dental acrylic. Acute imaging was performed an hour after the surgery; chronic imaging happened at least one week after the surgery. Mice were head-fixed and anesthetized using isoflurane (1-2\% by volume in 02$)$ during imaging.

Some mice also underwent virus injection procedures as described previously ${ }^{31}$. For the examples shown in Supplementary Fig. 9, neurons in the hippocampus were infected with a mixture of AAV-Syn-Cre (10x dilution from $\left.1.8 \times 10^{13} \mathrm{GC} / \mathrm{mL}\right)$ and AAV-CAG-FLEX-tdTomato $\left(3.3 \times 10^{13} \mathrm{GC} / \mathrm{mL}\right)$ in a wild-type mouse (C57BL/6J). Three injection sites were chosen (AP: $-1.7 \mathrm{~mm}, \mathrm{ML}:+1.5 \mathrm{~mm}$; AP: $-2.0 \mathrm{~mm}$, ML: +2.0 mm; AP: $2.3 \mathrm{~mm}, \mathrm{ML}:+2.5 \mathrm{~mm})$, at five different depths $(0.6,0.8,1,1.2$, and $1.4 \mu \mathrm{m}) .100 \mathrm{~nL}$ of viral solution were injected at each spot. A cranial window was implanted, as described above, 14 days after virus injection and acute imaging was then performed. 
Mouse preparation (spinal cord imaging). Acute spinal cord windows were prepared as described previously ${ }^{12}$. Briefly, mice were anesthetized with two successive intraperitoneal injections of $1 \mathrm{mg} / \mathrm{kg}$ body weight urethane each, 30 mins apart. A tracheotomy was performed to prevent asphyxiation. The T11-13 vertebrae were exposed and stabilized using spinal clamps (STS-A, Narishige). A dorsal laminectomy was performed at T12 to expose the spinal cord. After a wash with Ringer solution $(135 \mathrm{mM} \mathrm{NaCl}, 5.4 \mathrm{mM} \mathrm{KCl}, 5$ mM HEPES, $1.8 \mathrm{mM} \mathrm{CaCl}_{2}, \mathrm{pH}$ 7.2), the spinal cord was covered with a glass window made of a single coverslip (Fisher Scientific No. 1.5). The window and the surrounding custom imaging chamber were stabilized using $2 \%$ agarose in Ringer solution. Blood flow through the central blood vessel was continuously monitored throughout the imaging experiment to ensure tissue health. For the functional imaging experiments, wild-type (C57BL/6J) mice had previously been intrathecally injected with $\sim 5 \times 10^{10} \mathrm{GC}$ of AAV8-Syn-jGCaMP7s.

Temperature stimulation (spinal cord imaging): The left hind limb of the mouse was dehaired and gently fixed inside a custom-designed stimulation device as previously described ${ }^{12}$. The entire limb was continuously exposed to a homogeneous high flow-rate water flow of variable temperature. For each trial, the spinal cord was imaged for a total of $70 \mathrm{~s}$. The first $\sim 10 \mathrm{~s}$ were imaged at $31^{\circ} \mathrm{C}$ baseline water to obtain the baseline fluorescence and noise. For the following $\sim 30 \mathrm{~s}$ the flow was switched to water that was pre-incubated at colder temperatures with the same flow rate. For the remaining $\sim 30$ s the flow was switched back to the $31^{\circ} \mathrm{C}$ baseline water. At least another $120 \mathrm{~s}$ passed before the next trial was performed. The actual temperature inside the stimulation chamber was monitored and recorded using a microprobe thermometer (BAT-12, Physitemp) with a Type-K thermocouple placed right next to the mouse's limb, simultaneously with the fluorescence images. The electric valves controlling the water flow switch were triggered by and synchronized with the image acquisition system.

Analysis of calcium imaging data. To account for motion in the spinal cord caused by respiration, we processed the image sequences using an iterative cross-correlation-based registration algorithm ${ }^{31}$. We averaged across trials, manually selected the region of interest, and calculated the mean fluorescence within this region. From this, we calculated the fractional change in $3 \mathrm{P}$ fluorescence $\left(\Delta F / F_{0}\right)$ due to neural activity, with $F_{0}$ being the baseline fluorescence calculated as the mean fluorescence during exposure to the baseline temperature (initial $\sim 10 \mathrm{~s}$ of the temperature stimulation). For the traces shown in Fig. 3j, we performed a 5frame moving average.

\section{Supplementary Videos}

The data presented here did not undergo any smoothing or any other digital manipulation, except for image registration.

Supplementary Video 1: In vivo image stacks of cortical neuron in Thy1-YFP-H mouse measured without and with AO. Same data as shown in Fig. 2a. Scale bar, $10 \mu \mathrm{m}$.

Supplementary Video 2: Zoomed-in views of in vivo image stacks of cortical neuron dendrites in Thy1-YFP-H mouse measured without and with AO. Same data as shown in Fig. 2b. Scale bar, $10 \mu \mathrm{m}$.

Supplementary Video 3: In vivo image stacks of cortical neuron in Thy1-YFP-H mouse measured without and with AO. Same data as shown in Supplementary Fig. 6a. Scale bar, $10 \mu \mathrm{m}$.

Supplementary Video 4: Zoomed-in views of in vivo image stacks of cortical neuronal processes with dendritic spines and axonal boutons in Thy1-YFP-H mouse measured without and with AO. Same data as shown in Supplementary Fig. 6d. Scale bar, $10 \mu \mathrm{m}$.

Supplementary Video 5: Stack of 3P fluorescence and third-harmonic images going from cortex to the hippocampus of a Thy1-YFP-H mouse brain in vivo. This image sequence of combined $3 \mathrm{P}$ fluorescence and thirdharmonic signals was obtained from $250 \mu \mathrm{m}$ to $754 \mu \mathrm{m}$ below the dura of a mouse whose hippocampal neurons are shown in Fig. 2e-h Note the transition from cortex to hippocampus, through the white matter. Scale bar, $20 \mu \mathrm{m}$.

Supplementary Video 6: In vivo image stacks of hippocampal neuron in Thy1-YFP-H mouse measured without and with AO. Same data as shown in Fig. 2e. Scale bar, $10 \mu \mathrm{m}$.

Supplementary Video 7: Zoomed-in views of in vivo image stacks of hippocampal neuronal processes in Thy1YFP-H mouse measured without and with AO. Same data as shown in Fig. 2f. Scale bar, $10 \mu \mathrm{m}$.

Supplementary Video 8: In vivo image stacks of hippocampal neurons in Gad2-IRES-Cre × Ai14 (Rosa26-CAG-LSLtdTomato) mouse measured without and with AO. Same data as shown in Fig. 2i. Scale bar, $10 \mu \mathrm{m}$.

Supplementary Video 9: AO improves calcium activity recordings in the mouse spinal cord in vivo, $310 \mu \mathrm{m}$ below dura. Image sequence corresponding to data shown in Fig. 3i-k (4-trial average). Scale bar, $10 \mu \mathrm{m}$. 


\section{Figures}

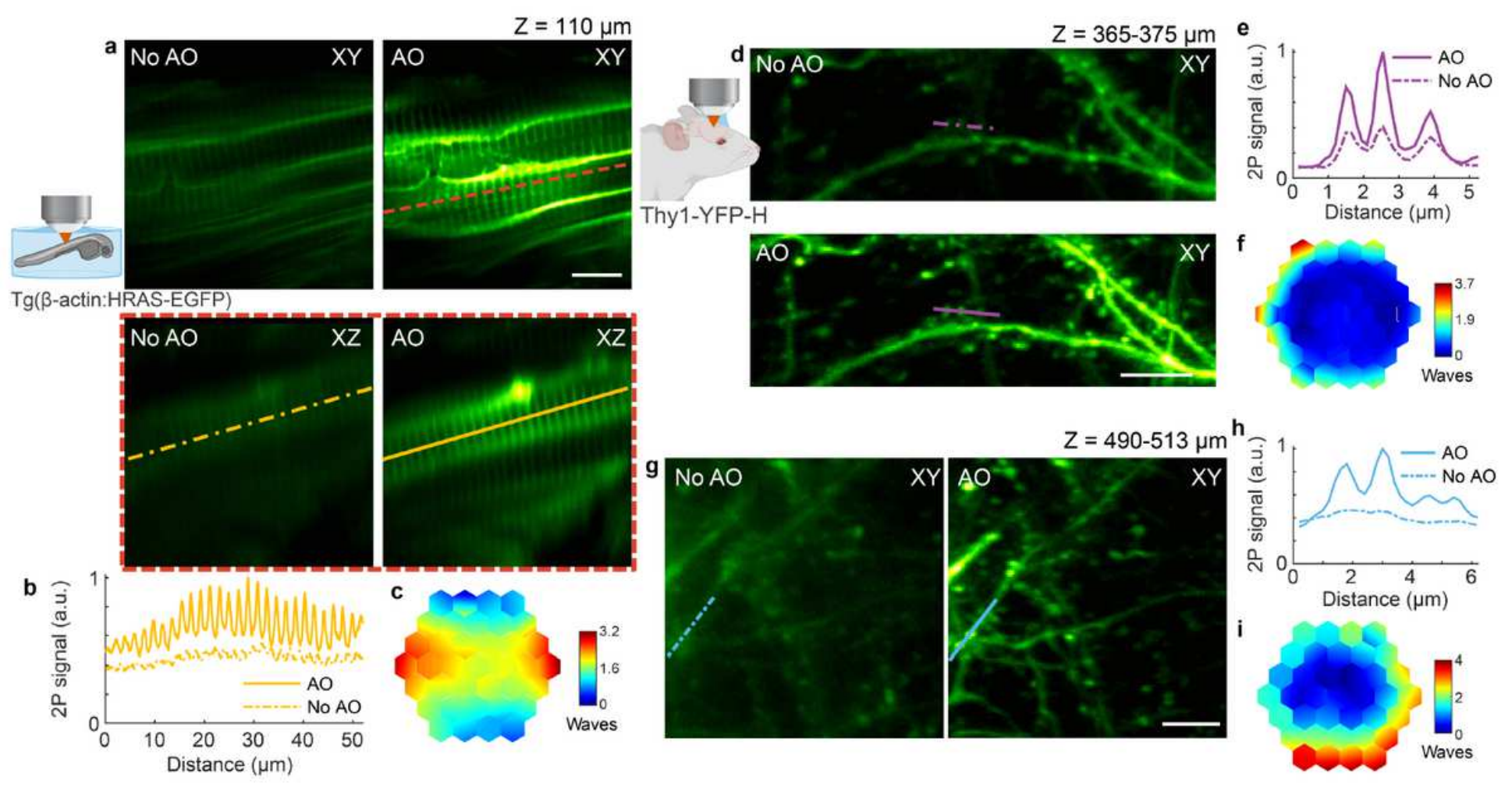

\section{Figure 1}

$\mathrm{AO}$ improves in vivo $2 \mathrm{P}$ imaging of myotomes in zebrafish larva and neuronal structures in the mouse brain. a, Lateral and axial (along red dashed line) images of myotomes in the mid-trunk of a 4-day-old zebrafish larva, at an imaging depth of $110 \mu \mathrm{m}$ from the surface, without and with AO (phase modulation). Post-objective power: $13 \mathrm{~mW}$. b. Signal profiles in the axial plane along the yellow lines in a. c, Corrective wavefront in a. d,g, Maximum intensity projections of dendrites in the Thy1-YFP-H mouse cerebral cortex at 365-375 $\mu \mathrm{m}$ and 490-513 $\mu \mathrm{m}$ below dura, respectively, under $920 \mathrm{~nm}$ excitation, without and with $\mathrm{AO}$ (phase modulation). Post-objective power: 31 and $128 \mathrm{~mW}$, respectively. e,h, Signal profiles along the purple and blue lines in $d$ and $g$, respectively. $f, i$, Corrective wavefronts in $d$ and $g$, respectively. Scale bars, $10 \mu \mathrm{m}$ in a and $5 \mu \mathrm{m}$ in $\mathrm{d}$ and g. Microscope objective: NA $0.816 \times$ for a and NA $1.0525 \times$ for $\mathrm{d}$ and $\mathrm{g}$. 


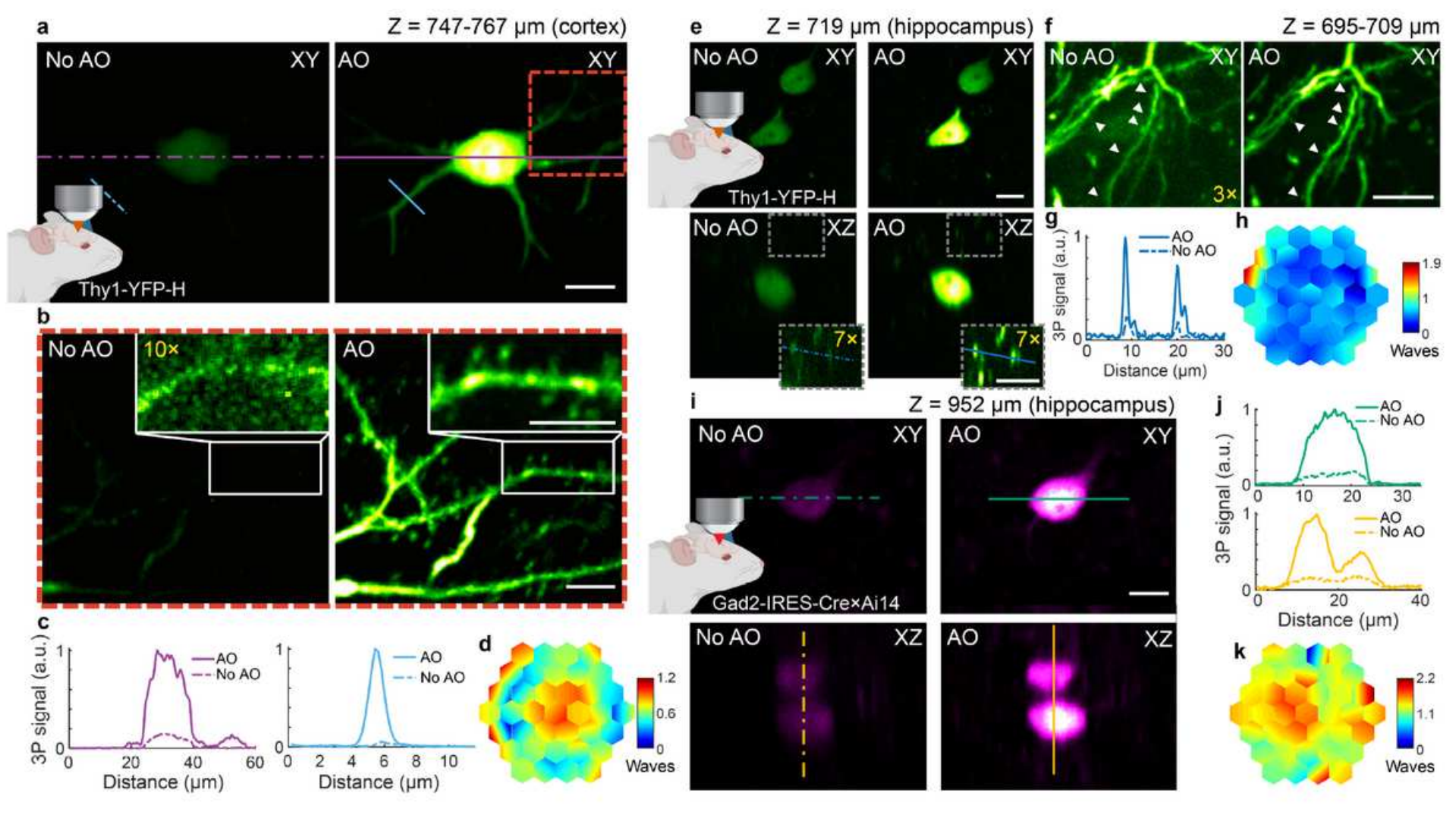

Figure 2

$\mathrm{AO}$ enables in vivo $3 \mathrm{P}$ imaging of cortical and hippocampal neuronal structures in the mouse brain, with subcellular resolution. a, Maximum intensity projection (MIP) of a neuron in the mouse cortex (Thy1-YFP$\mathrm{H})$, at 747-767 $\mu \mathrm{m}$ below dura, under $1300 \mathrm{~nm}$ excitation, without and with $\mathrm{AO}$ (phase modulation). Postobjective power: $17 \mathrm{~mW}$. b, Zoomed-in views of the red square in a, at 751-767 $\mu \mathrm{m}$ below dura, without and with AO. Insets, zoomed-in views of the dendrite in white rectangles in b. 10x digital gain was applied to No AO inset to increase visibility. Post-objective power: $20 \mathrm{~mW}$. c, Signal profiles along the purple and blue lines in a. $d$, Corrective wavefront in a and b. e, Lateral and axial images of neurons in the mouse hippocampus (Thy1-YFP-H), $719 \mu \mathrm{m}$ below dura, under $1300 \mathrm{~nm}$ excitation, without and with $\mathrm{AO}$ (phase modulation). Post-objective power: $16 \mathrm{~mW}$. Insets, zoomed-in views of the gray square in e. $7 \times$ digital gain was applied to increase visibility. f, MIP of neuronal processes above the cell body in e, at 695-709 $\mu \mathrm{m}$ below dura, without and with AO. White arrows: dendritic spines. Post-objective power: $26 \mathrm{~mW}$. $3 \times$ digital gain was applied to image without $A O$ to improve visibility. $g$, Signal profiles along the blue lines in e. h, Corrective wavefront in e and f. i, Lateral and axial images 

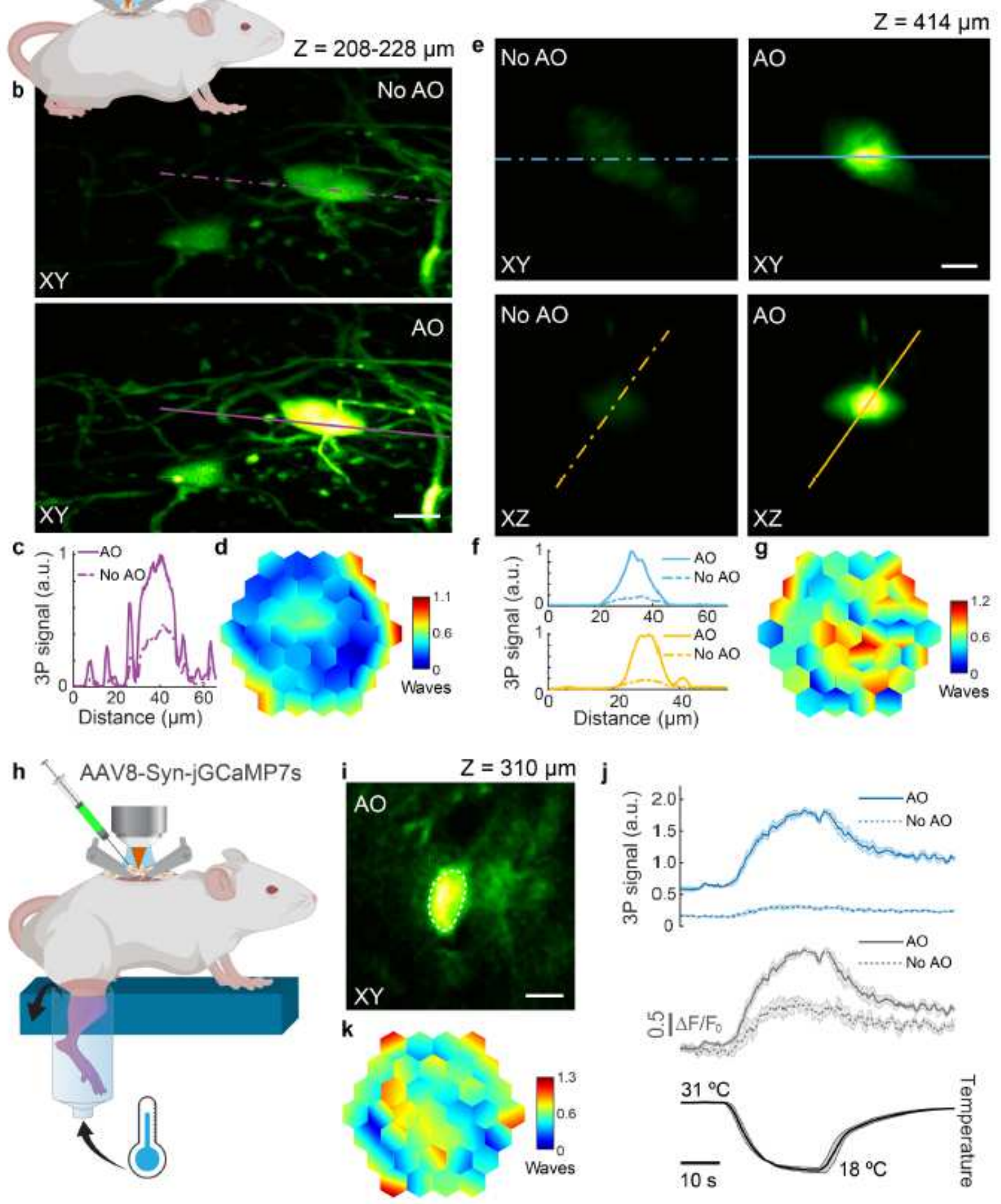

\section{Figure 3}

AO improves in vivo 3P structural and functional imaging in the mouse spinal cord. a, Schematic of in vivo imaging in the dorsal horn of the mouse spinal cord. b, Maximum intensity projection of spinal cord neurons (Thy1-GFP-M), 208-228 $\mu \mathrm{m}$ below dura, under $1300 \mathrm{~nm}$ excitation, without and with AO (phase modulation). Post-objective power: $18.3 \mathrm{~mW}$. c, Signal profiles along the purple lines in b. d, Corrective wavefront in b. e, Lateral and axial images of a neuron (Thy1-GFP-M), $414 \mu \mathrm{m}$ below dura, under 1300 
$\mathrm{nm}$ excitation, without and with $\mathrm{AO}$ (phase modulation). Post-objective power: $89 \mathrm{~mW}$. f, Signal profiles along the blue and yellow lines in e. g, Corrective wavefront in e. h, Schematic for recording calcium activity in jGCaMP7s-expressing neurons of the dorsal horn in the mouse spinal cord (AAV8-SynjGCaMP7s), in response to cooling stimuli applied to the skin of the hindlimb. i, Lateral image of a neuron, $310 \mu \mathrm{m}$ below dura, under $1300 \mathrm{~nm}$ excitation, after AO correction. j, (top) 3P fluorescence signal and (middle) calcium transients $(\triangle \mathrm{F} / \mathrm{FO})$ ), during (bottom) temperature stimulation, without and with $\mathrm{AO}$ (phase modulation), for the neuronal cell body shown in i. 4-trial average; shaded area: s.e.m. Postobjective power: $4.2 \mathrm{~mW}$. k, Corrective wavefront in i. Scale bars, $10 \mu \mathrm{m}$. Microscope objective: NA 1.05 $25 \times$.

\section{Supplementary Files}

This is a list of supplementary files associated with this preprint. Click to download.

- Video1.avi

- Video2.avi

- Video3.avi

- Video4.avi

- Video5.avi

- Video6.avi

- Video7.avi

- Video8.avi

- Video9.avi 